\title{
Desplazamiento cotidiano de estudiantes entre comunas de Chile: evidencia y recomendaciones de politica para la nueva institucionalidad de la Educación Pública
}

\author{
Sebastián Donoso-Díaz. Universidad de Talca, Talca, Chile. \\ Óscar Arias-Rojas. Universidad de Talca, Talca, Chile.
}

\begin{abstract}
RESUMEN | Una característica dominante del sistema escolar chileno es la discriminación en la oferta educativa, manifiesta en una fuerte asociación entre resultados de aprendizaje, por una parte, e ingreso y capital cultural de los hogares, por otra. Esta discriminación obliga a las familias a buscar establecimientos educacionales acordes a sus expectativas, generándose desplazamientos de estudiantes desde sus comunas de origen hacia otras localidades. A partir del análisis de la encuesta chilena de hogares del año 2006, se cuantifica este fenómeno (movilidad diaria de estudiantes), constatándose la existencia de patrones asociados a las dinámicas de poblamiento del territorio nacional: movilidad escasa en las regiones extremas, intermedia en las regiones del centro del país y alta en la Región Metropolitana de Santiago. De acuerdo con estos resultados, se discuten recomendaciones de política indispensables para incorporar la dimensión territorial en el diseño de la nueva institucionalidad de la educación pública en Chile.
\end{abstract}

PALABRAS CLAVE | movilidad, segregación, integración territorial.

ABSTRACT | A key feature of the Chilean school system is the discrimination in educational provision, showing a strong association between learning outcomes, on the one hand, and cultural capital and income of households, on the other. This discrimination forces families to seek educational institutions according to their expectations, generating mobility of students from their communities of origin to other communities. The analysis of the Chilean survey of households in 2006 allowed to quantify this phenomenon (student commuting), and demonstrated the existence of patterns associated with the dynamics of settlement of the population along the country: low mobility in the end regions, intermediate in the central regions of the country, and high in the Metropolitan Region of Santiago. According to these results, we discuss policy recommendations essential to incorporate the territorial dimension in the design of the new institutions of public education in Chile.

KEY WORDS | mobility, segregation, territorial integration.

Recibido el 6 de abril de 2011, aprobado el 1 de junio de 2012

E-mail: Sebastián Donoso, sdonoso@utalca.cl| Óscar Arias-Rojas, oshcar.arias@gmail.com

Instituto de Investigación y Desarrollo Educacional, Universidad de Talca, Chile. Este trabajo es también parte del Programa de Investigación Asociativa (PIA)-Comisión Nacional de Investigación Científica y Tecnológica (Conicyt)-Proyecto CIE 05 (Centro de Investigación Avanzada en Educación). 


\section{Antecedentes}

A pesar de los discretos pero sostenidos avances que ha mostrado en la última década el sistema educativo chileno en la prueba PISA (Programa Internacional de Evaluación de Estudiantes, por su sigla en inglés), dos de sus sellos distintivos se han mantenido: una importante brecha de resultados de aprendizajes con los países de mayor desarrollo, y un nivel significativo de discriminación - en términos de acceso y resultados - hacia los grupos de población tradicionalmente más excluidos de las oportunidades educativas y del trabajo.

A pesar de la complejidad de la situación señalada - y de sus efectos no solo sobre la dinámica productiva, sino también sobre la legitimidad de la convivencia democrática-, desde los años noventa los actores técnicos y políticos han sido reacios a introducir reformas de fondo en la institucionalidad educativa heredada de la dictadura, uno de los principales emblemas de la política de transformación autoritaria que se llevó a cabo durante los años ochenta en Chile (Donoso, 2006).

En el ámbito educativo, la transformación impulsada por el gobierno militar significó el paso desde un sistema de subsidio a la oferta en educación (el Estado financiaba los presupuestos históricos de los establecimientos públicos), a un modelo de subsidio a la demanda (se comienza a aplicar un subsidio por estudiante atendido). Por otro lado, se descentralizó la administración de los establecimientos públicos, pasando los aspectos administrativos y de gestión a depender del nivel local (municipalidades), mientras los aspectos técnico-pedagógicos continuaron bajo supervisión del Ministerio de Educación (Mineduc). Con esto se buscó traspasar poderes a las administraciones locales, al tiempo que se intentaba mejorar la eficiencia en el uso de los recursos mediante la competencia por estudiantes entre los establecimientos públicos (educación municipal), los establecimientos privados con subvención del Estado (educación particular subvencionada), y los establecimientos privados sin subvención (educación particular pagada) (Donoso \& Schmal, 2009). Este arreglo significó el rápido y sostenido aumento de la oferta privada con subvención estatal: mientras en 1981 el $81 \%$ de la matrícula era de dependencia municipal (pública), hacia 1997 esta solo abarcaba el 56\% de los estudiantes, y para el año 2006 no alcanzaba el 47\%. En el mismo periodo, la matrícula particular subvencionada pasó de 13\% a 49\% (Mineduc, 2008).

Por otro lado, además de la baja en la participación de la educación pública en el sistema educativo chileno, desde mediados de los noventa se registra un creciente proceso de segmentación de estudiantes de acuerdo con su origen social. La falta de regulación en la selección de estudiantes por parte de las escuelas, y la existencia hasta el año 2007 de un subsidio educacional (voucher) prácticamente fijo -independiente de las condiciones de educabilidad de los estudiantes-, estimuló a los establecimientos particulares con subvención del Estado a poner barreras y filtros a la entrada de los estudiantes con mayores dificultades de aprendizaje (es decir, los más pobres y vulnerables), debido a su mayor costo. En definitiva, estos solo tienen un acceso asegurado a los establecimientos municipales (públicos). Ello implica que, 
en Chile, el derecho a la educación solo lo garantizan los establecimientos públicos, pese a existir un importante sector privado financiado con recursos públicos.

De este modo, la oferta de educación pública se ha convertido crecientemente en un servicio de segunda mano, desprestigiado socialmente, pero no necesariamente menos eficiente: estudios recientes muestran que sus bajos resultados comparativos no se explican en forma concluyente por una mayor eficiencia de los establecimientos privados con subvención, sino porque la educación pública recibe a una población de mayor vulnerabilidad, a la que debe ofrecer educación con un aporte estatal similar al de la educación subvencionada y en un marco institucional más restringido en términos de autonomía, uso de recursos y posibilidades de gestión (Tokman, 2001; Hsieh \& Urquiola, 2002; Donoso \& Hawes, 2002; Mizala \& Romaguera, 2003; Treviño \& Donoso, 2010). El Cuadro 1 da cuenta de tres constantes del sistema escolar de los últimos años: i) la relación entre resultados educativos y grupo socioeconómico; ii) el traspaso de estudiantes de los sectores medios desde la educación pública hacia la particular subvencionada; y iii) la ausencia de diferencias importantes entre los resultados del sector público y del privado cuando se controla por origen social (incluso hay una tendencia a mejores resultados en los establecimientos públicos en el segmento de menores ingresos).

La mayor competencia entre establecimientos, impulsada expresamente por las políticas educacionales durante la dictadura, terminó de instalarse en las administraciones democráticas de los años noventa; al no contar la educación particular subvencionada con un subsidio diferenciado - calculado de acuerdo con las condiciones de educabilidad real de los estudiantes - ni con un sistema de regulación para la selección de estudiantes, se generó en la práctica una suerte de "competencia desleal” con los establecimientos públicos. Esto no solo produjo una creciente y sistemática pérdida de estudiantes en la educación pública. Al mismo tiempo, introdujo distorsiones severas en términos de calidad, pues al estar segmentados los establecimientos de acuerdo con el origen social e ingresos de los estudiantes y sus familias, el racional de elección de establecimientos por parte de las familias depende básicamente de la adscripción o aspiración a cierto estatus social, o de la búsqueda de prestigio y redes sociales, u otros tipos de bienestar, más que de los resultados de aprendizaje (Hsieh \& Urquiola, 2006; Treviño \& Donoso, 2010).

La reticencia de las autoridades para reconocer esta problemática y para proponer reformas de fondo, se vio desafiada por la crisis inducida por las manifestaciones estudiantiles del año 2006. Una de las demandas centrales de los manifestantes - que contaron con una amplia legitimidad y respaldo popular- fue la revisión completa de la institucionalidad educativa implementada por la dictadura (a partir de los años ochenta) y por los gobiernos democráticos (a partir de los noventa). Entre otras medidas tendientes a mejorar la calidad y equidad del sistema, el gobierno respondió con la constitución de un inédito Consejo Asesor Presidencial para la Calidad de la Educación, que recibió la tarea de "mostrar caminos, hacer recomendaciones y sugerencias para mejorar la calidad de la educación que se ofrece en escuelas y liceos del país a todos los niños, niñas y jóvenes, sin importar su origen social, económico y cultural" (Consejo Asesor, 2006, p. 12). 
CUADRO 1 Evolución de la proporción de estudiantes por nivel socioeconómico, dependencia educacional y resultados SIMCE en Matemáticas (4º́sico), $2002-2009$

\begin{tabular}{|c|c|c|c|c|c|c|c|c|c|}
\hline \multirow[t]{2}{*}{ Año } & \multirow[t]{2}{*}{ NSE } & \multicolumn{2}{|c|}{ Municipal } & \multicolumn{2}{|c|}{ Particular Subvencionado } & \multicolumn{2}{|c|}{ Particular Pagado } & \multicolumn{2}{|c|}{ Total } \\
\hline & & $\%$ & SIMCE & $\%$ & SIMCE & $\%$ & SIMCE & $\%$ & SIMCE \\
\hline \multirow[t]{6}{*}{2002} & Bajo & 8 & 223 & 2 & 210 & 0 & - & 10 & 220 \\
\hline & $\begin{array}{l}\text { Medio } \\
\text { Bajo }\end{array}$ & 26 & 229 & 6 & 227 & 0 & - & 31 & 229 \\
\hline & Medio & 18 & 246 & 20 & 253 & 0 & 258 & 37 & 250 \\
\hline & $\begin{array}{l}\text { Medio } \\
\text { Alto }\end{array}$ & 2 & 271 & 12 & 275 & 1 & 277 & 15 & 275 \\
\hline & Alto & 0 & - & 0 & 299 & 6 & 301 & 7 & 301 \\
\hline & TOTAL & 53 & 235 & 40 & 254 & 7 & 298 & 100 & 247 \\
\hline \multirow[t]{6}{*}{2005} & Bajo & 6 & 225 & 2 & 207 & 0 & - & 8 & 220 \\
\hline & $\begin{array}{l}\text { Medio } \\
\text { Bajo }\end{array}$ & 23 & 228 & 5 & 226 & 0 & - & 28 & 227 \\
\hline & Medio & 18 & 242 & 18 & 249 & 0 & 236 & 37 & 245 \\
\hline & $\begin{array}{l}\text { Medio } \\
\text { Alto }\end{array}$ & 2 & 267 & 18 & 272 & 0 & 264 & 20 & 271 \\
\hline & Alto & 0 & - & 1 & 290 & 6 & 299 & 7 & 298 \\
\hline & TOTAL & 49 & 235 & 44 & 255 & 6 & 297 & 100 & 248 \\
\hline \multirow[t]{6}{*}{2006} & Bajo & 6 & 224 & 2 & 206 & 0 & - & 8 & 220 \\
\hline & $\begin{array}{l}\text { Medio } \\
\text { Bajo }\end{array}$ & 23 & 227 & 6 & 227 & 0 & - & 29 & 227 \\
\hline & Medio & 17 & 243 & 21 & 252 & 0 & 222 & 37 & 248 \\
\hline & $\begin{array}{l}\text { Medio } \\
\text { Alto }\end{array}$ & 2 & 268 & 17 & 272 & 0 & 263 & 18 & 272 \\
\hline & Alto & 0 & - & 1 & 288 & 6 & 298 & 7 & 297 \\
\hline & TOTAL & 48 & 234 & 46 & 255 & 7 & 297 & 100 & 248 \\
\hline \multirow[t]{6}{*}{2007} & Bajo & 7 & 224 & 2 & 210 & 0 & - & 9 & 221 \\
\hline & $\begin{array}{l}\text { Medio } \\
\text { Bajo }\end{array}$ & 24 & 225 & 7 & 229 & 0 & - & 30 & 226 \\
\hline & Medio & 14 & 240 & 22 & 250 & 0 & - & 36 & 246 \\
\hline & $\begin{array}{l}\text { Medio } \\
\text { Alto }\end{array}$ & 1 & 269 & 15 & 273 & 0 & 265 & 17 & 273 \\
\hline & Alto & 0 & - & 1 & 291 & 6 & 299 & 7 & 298 \\
\hline & TOTAL & 46 & 230 & 47 & 254 & 7 & 298 & 100 & 246 \\
\hline \multirow[t]{6}{*}{2008} & Bajo & 8 & 223 & 2 & 209 & 0 & - & 10 & 220 \\
\hline & $\begin{array}{l}\text { Medio } \\
\text { Bajo }\end{array}$ & 23 & 226 & 6 & 227 & 0 & - & 29 & 227 \\
\hline & Medio & 13 & 241 & 21 & 249 & 0 & 264 & 34 & 246 \\
\hline & $\begin{array}{l}\text { Medio } \\
\text { Alto }\end{array}$ & 2 & 266 & 17 & 273 & 0 & 262 & 19 & 272 \\
\hline & Alto & 0 & - & 1 & 289 & 7 & 302 & 8 & 300 \\
\hline & TOTAL & 45 & 231 & 48 & 254 & 7 & 301 & 100 & 247 \\
\hline \multirow[t]{6}{*}{2009} & Bajo & 8 & 226 & 2 & 216 & 0 & - & 10 & 224 \\
\hline & $\begin{array}{l}\text { Medio } \\
\text { Bajo }\end{array}$ & 23 & 233 & 8 & 236 & 0 & - & 31 & 234 \\
\hline & Medio & 11 & 247 & 25 & 258 & 0 & - & 36 & 255 \\
\hline & $\begin{array}{l}\text { Medio } \\
\text { Alto }\end{array}$ & 1 & 278 & 15 & 279 & 0 & - & 16 & 279 \\
\hline & Alto & 0 & - & 1 & 299 & 7 & 304 & 8 & 304 \\
\hline & TOTAL & 43 & 236 & 50 & 259 & 7 & 303 & 100 & 253 \\
\hline
\end{tabular}

NOTA 1 SD= Sin Datos. Porcentajes APROXIMAdos (PODRÍAN NO SUMAR 100\%).

NOTA 2 DESCRIPCIÓN DE LOS INTERVALOS DE CLASIFICACIÓN SOCIOECONÓMICA (SIMCE 4º BÁSICO 2009): 


\begin{tabular}{|c|c|c|c|c|}
\hline \multirow[t]{2}{*}{ GRUPO } & \multicolumn{2}{|c|}{ ESCOLARIDAD } & \multirow[t]{2}{*}{$\begin{array}{l}\text { INGRESO DEL } \\
\text { HOGAR }\end{array}$} & \multirow{2}{*}{$\begin{array}{c}\text { ÍNDICE DE } \\
\text { VULNERABILIDAD DEL } \\
\text { ESTABLECIMIENTO }\end{array}$} \\
\hline & Madre & Padre & & \\
\hline Bajo & Menos de 9 años & Menos de 9 años & Hasta $\$ 152.000$ & Más de $80 \%$ \\
\hline Medio Bajo & Entre 9 y 10 años & Entre 9 y 10 años & $\begin{array}{l}\text { Entre } \$ 152.001 \text { y } \\
\quad \$ 230.000\end{array}$ & Entre $50,01 \%$ y $80 \%$ \\
\hline Medio & Entre 11 y 12 años & Entre 11 y 12 años & $\begin{array}{l}\text { Entre } \$ 230.001 \mathrm{y} \\
\quad \$ 400.000\end{array}$ & Entre $25,01 \%$ y $50 \%$ \\
\hline Medio Alto & Entre 13 y 15 años & Entre 13 y 15 años & $\begin{array}{c}\text { Entre } \$ 400.001 \mathrm{y} \\
\quad \$ 1.100 .00\end{array}$ & Entre $5,01 \%$ y $50 \%$ \\
\hline Alto & Más de 15 años & Más de 15 años & Más de $\$ 1.100 .000$ & Entre $0 \%$ y $5 \%$ \\
\hline
\end{tabular}

FUENTE ELABORACIÓN PROPIA A PARTIR DE SIMCE (2009).

Respecto de los bajos resultados de la educación municipal, junto con destacar los problemas de financiamiento, de gestión y de competencia desigual con el sistema particular subvencionado, los consejeros mencionaron como un factor negativo decisivo la diversidad de población de las comunas y sus diferentes capacidades de gestión (Consejo Asesor, 2006, p. 26). En efecto, si se comparan las condiciones territoriales y de gestión que enfrentan los encargados de administrar la educación pública, se establecen diferencias importantes (Cuadro 2): tres cuartas partes de la población escolar del país se concentra en las 86 comunas más pobladas (de un total de 345 comunas).

Las comunas menos pobladas son también las que tienen mayores niveles de ruralidad y pobreza. $\mathrm{Al}$ administrar cada comuna un sistema de educación pública propio, sin conexiones formales con los sistemas aledaños, con una subvención insuficiente y sin criterios operativos que relacionen sus acciones con el desarrollo del territorio local, las diferencias tienden a reproducirse. El 50\% de las comunas menos pobladas debe gestionar sistemas con muy pocos estudiantes: menos de 2.500 en promedio, una cantidad que - en términos equivalentes - puede encontrarse en un solo establecimiento de una comuna urbana de gran población. Por otro lado, los habitantes de estos sistemas tienden a dispersarse más en el territorio, lo que implica establecimientos más pequeños y una menor tasa de estudiantes por docente, con el consiguiente aumento de los costos. A pesar de los mayores niveles de subvención en las comunas de menos habitantes, estas, que también son las pobres, deben realizar un significativo aporte por estudiante, lo que revela que la subvención está lejos de actuar como un criterio de compensación adecuado frente a las diferencias de origen social y territorial. La mayor participación de la matrícula municipal en las comunas de menor población refuerza la evidencia de que el subsidio no tiende a la igualdad: los costos de los oferentes privados no alcanzan a ser cubiertos por la subvención en zonas de baja población, lo que disminuye su presencia 
(Sapelli, 2006) y, por lo mismo, no se nivelan las oportunidades de los estudiantes más carentes (Roemer, 1998). A su vez, la distribución de esta diversidad local no es homogénea en el territorio nacional (Cuadro 3), lo que se refleja en la existencia de zonas diferenciadas cuando se las compara en el nivel subnacional. Se tiene así:

a) Regiones de poblamiento escaso, baja ruralidad, concentración en pocos centros urbanos (típicamente las capitales regionales), existencia de focos urbanos dispersos (Arica y Parinacota, Tarapacá, Antofagasta, Atacama, Aysén, Magallanes);

b) Regiones de poblamiento intermedio, con una proporción importante de población localizada en zonas rurales, mayor densidad poblacional y menor concentración en comunas "centrales". Se trata de las regiones donde se concentra la población que vive en comunas de pocos habitantes (Coquimbo, O’Higgins, Maule, Araucanía, Los Ríos, Los Lagos); y

c) Regiones de mayor población, que incluyen zonas metropolitanas en torno a las capitales regionales (las cuales representan una baja proporción de la población), menores niveles de ruralidad y con más habitantes localizados en concentraciones urbanas importantes (Valparaíso, Santiago, Biobío).

La diversidad de contextos locales en que se administra la educación pública, unida a la existencia de sistemas muy pequeños, derivó en la recomendación de generar nuevas entidades de administración de la educación pública, contenida en el Informe del Consejo Asesor Presidencial para la Calidad de la Educación (2006). Aunque los consejeros no lograron consenso al respecto, distinguieron al menos tres alternativas: i) mantención de la administración local mediante los municipios e impulso a las asociaciones de municipios; ii) administración a nivel subnacional, con servicios provinciales o regionales de educación; y iii) un sistema de administración nacional centralizado en el Ministerio de Educación. Más allá del diagnóstico de las diferencias que enfrentan los distintos administradores locales de educación pública — en términos de gasto y de condiciones de gestión-, existe poca evidencia empírica respecto de las interacciones entre los administradores de la educación pública en un nivel superior al local, aspecto clave en cualquier propuesta que busque racionalizar, en un territorio mayor que el de las comunas, la provisión de educación pública. Esto es relevante, ya que la institucionalidad actual permite que las familias elijan escuelas y liceos más allá de las fronteras de su comuna de residencia. Tomando en consideración el proceso de pérdida de estudiantes de la educación pública a favor de la particular subvencionada, resulta relevante analizar cómo interactúan los sistemas educativos de distintas comunas en el principal desafío que les impone el esquema de vouchers: la competencia por estudiantes. En el presente trabajo se analizan en forma exploratoria los flujos cotidianos de movilidad regular de estudiantes entre comunas, y con base en esta evidencia empírica, se entregan recomendaciones de política para la nueva institucionalidad de la educación pública chilena. El examen de este fenómeno reporta evidencia importante para prever sus impactos negativos, debido a la tendencia privatizadora actual de la educación pública en los países emergentes. 
CUADRO 2 Comunas de Chile agrupadas según población e indicadores de caracterización comunal y de los sistemas educativos municipales (2007)

\begin{tabular}{|c|c|c|c|c|c|c|}
\hline \multirow[b]{2}{*}{ INDICADORES } & \multicolumn{5}{|c|}{ Comunas de Chile (según población) } & \multirow[b]{2}{*}{ TOTAL } \\
\hline & $\begin{array}{l}\text { Menos de } \\
10.000 \\
\text { habitantes }\end{array}$ & $\begin{array}{l}\text { Entre } 10.000 \\
\text { y } 19.999 \\
\text { habitantes }\end{array}$ & $\begin{array}{c}\text { Entre } 20.000 \\
\text { y } 49.999 \\
\text { habitantes }\end{array}$ & $\begin{array}{c}\text { Entre } 50.000 \\
\text { y } 149.999 \\
\text { habitantes }\end{array}$ & $\begin{array}{l}150.000 \\
\text { habitantes } \\
\text { y más }\end{array}$ & \\
\hline \multicolumn{7}{|l|}{ CARACTERIZACIÓN COMUNAL } \\
\hline Comunas I (total) & 99 & 87 & 73 & 56 & 30 & 345 \\
\hline Comunas II (\% del total) & $28,7 \%$ & $25,2 \%$ & $21,2 \%$ & $16,2 \%$ & $8,7 \%$ & $100,0 \%$ \\
\hline Población I (total) & 535.549 & 1.238 .138 & 2.218 .404 & 5.041 .992 & 7.563 .889 & 16.597 .972 \\
\hline Población II (\% del país) & $3,2 \%$ & $7,5 \%$ & $13,4 \%$ & $30,4 \%$ & $45,6 \%$ & $100,0 \%$ \\
\hline Ruralidad (promedio) & $61,7 \%$ & $46,4 \%$ & $32,8 \%$ & $8,9 \%$ & $3,7 \%$ & $38,1 \%$ \\
\hline $\begin{array}{l}\text { Población en situación de } \\
\text { pobreza (promedio) }\end{array}$ & $15,4 \%$ & $17,8 \%$ & $18,3 \%$ & $13,6 \%$ & $12,4 \%$ & $16,1 \%$ \\
\hline $\begin{array}{l}\text { Disponibilidad presupuestaria } \\
\text { municipal por habitante } \\
(\mathrm{M} \$ \text { al año) }\end{array}$ & $\$ 348$ & $\$ 118$ & $\$ 89$ & $\$ 99$ & $\$ 104$ & $\$ 174$ \\
\hline \multicolumn{7}{|c|}{ CARACTERIZACIÓN SISTEMAS EDUCATIVOS MUNICIPALES } \\
\hline Matrícula total (promedio) & 979 & 2.518 & 5.913 & 18.016 & 47.393 & 9.213 \\
\hline $\begin{array}{l}\text { Estudiantes por } \\
\text { establecimiento (promedio) }\end{array}$ & 132 & 191 & 259 & 522 & 650 & 282 \\
\hline $\begin{array}{l}\text { Estudiantes por docente de } \\
\text { aula (promedio) }\end{array}$ & 18 & 21 & 24 & 26 & 27 & 22 \\
\hline $\begin{array}{l}\text { Matrícula municipal respecto } \\
\text { de la matrícula comunal total } \\
\text { (promedio) }\end{array}$ & $90,2 \%$ & $76,6 \%$ & $62,8 \%$ & $43,0 \%$ & $40,8 \%$ & $69,0 \%$ \\
\hline \multicolumn{7}{|c|}{ GASTO EN EDUCACIÓN MUNICIPAL } \\
\hline $\begin{array}{l}\text { Aporte subvención escolar por } \\
\text { estudiante ( } \mathrm{M} \$ \text { al año) }\end{array}$ & $\$ 896$ & $\$ 611$ & $\$ 582$ & $\$ 488$ & $\$ 475$ & $\$ 654$ \\
\hline $\begin{array}{l}\text { Aporte municipal por } \\
\text { estudiante ( } \mathrm{M} \$ \text { al año) }\end{array}$ & $\$ 286$ & $\$ 92$ & $\$ 67$ & $\$ 117$ & $\$ 89$ & $\$ 146$ \\
\hline $\begin{array}{l}\text { Gasto total educación } \\
\text { municipal por estudiante } \\
(\mathrm{M} \$ \text { al año) }\end{array}$ & $\$ 1.344$ & $\$ 713$ & $\$ 565$ & $\$ 352$ & $\$ 314$ & $\$ 768$ \\
\hline
\end{tabular}

fuente Sistema Nacional de Información Municipal (Sinim) 2007 (pesos chilenos de 2007). 
CUADro 3 Diversidad de poblamiento de las regiones de Chile (2007)

\begin{tabular}{|c|c|c|c|c|c|c|c|}
\hline & $\begin{array}{c}\text { Población } \\
\text { regional }\end{array}$ & $\begin{array}{c}\text { Densidad } \\
\text { poblacional } \\
\left(\mathrm{hab} / \mathrm{km}^{2}\right)\end{array}$ & $\begin{array}{l}\text { Población } \\
\text { que vive en } \\
\text { capitales } \\
\text { regionales }\end{array}$ & $\begin{array}{c}\text { Población } \\
\text { que vive en } \\
\text { capitales } \\
\text { provinciales y } \\
\text { regionales }\end{array}$ & $\begin{array}{l}\text { Población } \\
\text { rural }\end{array}$ & $\begin{array}{c}\text { Población } \\
\text { que vive en } \\
\text { comunas de } \\
\text { más de } 50 \mathrm{mil} \\
\text { habitantes }\end{array}$ & $\begin{array}{c}\text { Población que } \\
\text { vive en comunas } \\
\text { de menos de } 10 \\
\text { mil habitantes }\end{array}$ \\
\hline $\begin{array}{l}\text { ARICA Y } \\
\text { PARINACOTA }\end{array}$ & 188.524 & 11,2 & $97,8 \%$ & $98,5 \%$ & $8,5 \%$ & $97,8 \%$ & $2,2 \%$ \\
\hline TARAPACÁ & 293.205 & 6,9 & $62,3 \%$ & $67,4 \%$ & $6,2 \%$ & $88,5 \%$ & $2,0 \%$ \\
\hline ANTOFAGASTA & 554.773 & 4,4 & $62,8 \%$ & $93,3 \%$ & $2,4 \%$ & $89,3 \%$ & $2,9 \%$ \\
\hline ATACAMA & 274.436 & 3,7 & $55,5 \%$ & $77,6 \%$ & $9,0 \%$ & $55,5 \%$ & $6,8 \%$ \\
\hline COQUimbo & 687.659 & 17 & $28,4 \%$ & $48,7 \%$ & $20,2 \%$ & $72,8 \%$ & $4,5 \%$ \\
\hline VALPARAÍSO & 1.701 .293 & 103,8 & $16,2 \%$ & $37,6 \%$ & $8,5 \%$ & $71,3 \%$ & $3,5 \%$ \\
\hline SANTIAGO & 6.676 .745 & 433,5 & $2,7 \%$ & $20,9 \%$ & $3,1 \%$ & $96,5 \%$ & $0,2 \%$ \\
\hline o'HIGGINS & 857.677 & 52,3 & $27,7 \%$ & $37,5 \%$ & $29,3 \%$ & $42,6 \%$ & $6,4 \%$ \\
\hline MAULE & 983.396 & 32,5 & $23,5 \%$ & $50,5 \%$ & $33,1 \%$ & $51,5 \%$ & $6,8 \%$ \\
\hline Bío Bío & 1.996 .099 & 53,9 & $11,3 \%$ & $30,8 \%$ & $16,9 \%$ & $65,7 \%$ & $4,2 \%$ \\
\hline ARAUCANÍA & 945.544 & 29,7 & $30,4 \%$ & $35,8 \%$ & $32,1 \%$ & $48,9 \%$ & $4,7 \%$ \\
\hline LOS RÍOS & 375.193 & 20,4 & $41,4 \%$ & $51,9 \%$ & $31,1 \%$ & $41,4 \%$ & $5,9 \%$ \\
\hline LOS LAGOS & 804.975 & 16,6 & $27,2 \%$ & $53,9 \%$ & $30,3 \%$ & $47,0 \%$ & $10,2 \%$ \\
\hline AYSÉN & 101.523 & 0,9 & $55,2 \%$ & $87,9 \%$ & $16,7 \%$ & $55,2 \%$ & $19,8 \%$ \\
\hline MAGALLANES & 156.930 & 1,2 & $79,0 \%$ & $97,3 \%$ & $7,1 \%$ & $79,0 \%$ & $7,9 \%$ \\
\hline CHILE & 16.597 .972 & 22 & $18,4 \%$ & $36,9 \%$ & $13,1 \%$ & $75,9 \%$ & $3,2 \%$ \\
\hline
\end{tabular}

$\left(^{*}\right)$ CONSIDERA EDUCACIÓN MUNICIPAL Y EDUCACIÓN PARTICULAR SUBVENCIONADA. FUENTE SINIM 2007

\section{Metodología}

Para el análisis de la movilidad o desplazamiento de matrícula se utilizó la base de la Encuesta de Caracterización Socioeconómica Nacional (Casen) y la información censal del directorio de establecimientos educativos y estudiantes matriculados del Ministerio de Educación de Chile (Mineduc), tomándose como referencia el año 
2006. El concepto de movilidad cotidiana (o regular) que se emplea abarca los traslados diarios de estudiantes entre hogares y establecimientos que se encuentran en comunas diferentes. Se filtraron del análisis los traslados a distancias superiores a los 300 kilómetros diarios (ida y vuelta), porque responden a otra dinámica. Se consideró la información de los establecimientos municipales y particulares subvencionados, en los niveles de educación básica y educación media. El análisis que se presenta es de tipo transversal, de modo que se estudió la movilidad de estudiantes al interior de cada sistema - según tipo de educación (municipal/particular subvencionada) y nivel de enseñanza (básica/media)—, pero no entre sistemas. A pesar de que este tipo de análisis permite conocer características relevantes sobre la movilidad cotidiana, la revisión exhaustiva del fenómeno entre el sistema municipal y el particular subvencionado requiere un análisis longitudinal de cohortes.

No se incluye el análisis de la educación particular pagada, primero porque ella no se vincula con la nueva arquitectura de la educación pública; segundo, porque atañe a un segmento menor de la población, inferior al 8\% de los estudiantes; además, porque opera con criterios de funcionamiento muy diferentes a los del sector financiado por el Estado; y finalmente, por la inexistencia de esta oferta en la mayoría de las comunas del país.

Para la caracterización comunal y regional se utilizó la información del Sistema Nacional de Información Municipal (Sinim) del año 2007. La principal limitación del estudio se asocia a la baja cantidad de casos en las comunas de menor población, y al hecho de que la encuesta Casen 2006 no distingue lugar de residencia permanente o casual, ni tampoco ofrece referencias geográficas para el interior de las comunas. En este sentido, este trabajo debe considerarse como una exploración de tendencias generales de una temática no estudiada en Chile, que analiza los traslados de estudiantes entre comunas como una variable relevante para el diseño de una nueva arquitectura del sistema de educación pública. Tal exploración deberá ser confirmada por un análisis con información censal ${ }^{1}$ de estudiantes que provea información a un nivel de agregación inferior al comunal.

\section{Resultados}

\section{Movilidad de estudiantes en el sistema escolar y patrones de movilidad diaria regional}

Los Cuadros 4 y 5 dan cuenta de un conjunto importante de información: en primer lugar, a nivel nacional la movilidad regular de estudiantes en educación media es mayor (alcanza el 31\%) que la de educación básica (11\%). Segundo, en ambos niveles de enseñanza la movilidad es mayor entre los estudiantes del sistema particular subvencionado que en el municipal. Tercero, a pesar de ello, al comparar los diversos tipos de educación, se aprecian con claridad tres grupos de regiones: las de escasa movilidad regular (Arica y Parinacota, Antofagasta, Atacama, Aysén, Ma-

1 Se utiliza el término 'censal' como cualquier tipo de recopilación de datos de carácter censal, es decir, que entrega información sobre cada una de las unidades del universo. 
gallanes), las regiones de movilidad intermedia (Tarapacá, Coquimbo, Valparaíso, O’Higgins, Maule, Biobío, Araucanía, Los Ríos y Los Lagos) y una región de alta movilidad diaria (Santiago).

Respecto de la menor movilidad en educación básica, ella puede explicarse por el hecho de tratarse de un segmento en el cual la universalidad territorial de la enseñanza está consolidada. Esto da lugar a una oferta de mayor homogeneidad en más comunas, de modo que los incentivos a las familias para buscar establecimientos más lejanos son menores. Lo contrario sucede en educación media: el aumento en la cobertura - que falta para que sea universal-, se sustenta en una oferta de mayor heterogeneidad tanto en su orientación como en antigüedad, dando origen a mayores diferencias de resultados entre los establecimientos.

En razón de lo expuesto, en la enseñanza secundaria se aprecia con mayor nitidez los efectos de la segregación de los establecimientos en materia de resultados, agregándose a la diversidad de la oferta (Científico Humanista o Técnico Profesional con distintas especialidades), la edad de los estudiantes, que es también una variable asociada a la movilidad: mientras más pequeños, es más probable que asistan a un estableciendo más cercano al hogar. De este modo, hay varios elementos en el nivel de educación media que pueden incentivar a un mayor número de familias a buscar establecimientos fuera de la comuna de residencia.

Adicionalmente, para una mejor comprensión de la temática, la mayor movilidad regular en educación particular subvencionada se relaciona con la menor presencia de esta oferta en los territorios de menor desarrollo y población y de mayor aislamiento, expuestos en el Cuadro 2, los que muestran cómo disminuye la participación de la educación particular subvencionada en dichos territorios, ya que se trata de un tipo de educación que atiende a población de mayores ingresos. Así, en territorios pobres o aislados, los estudiantes provenientes de hogares con mejores ingresos muestran mayor disposición a estudiar fuera de la comuna de residencia habitual, impulsados por sus mayores expectativas, su capacidad de elegir establecimientos y sus posibilidades de desplazamiento.

Además de las diferencias generales, en la movilidad por tipo de establecimiento y nivel de enseñanza resulta relevante la relación entre los patrones de desplazamiento a escala regional y la caracterización de las regiones, expuestos precedentemente en el Cuadro 3, destacando que los niveles de movilidad de estudiantes, especialmente en educación media, están relacionados con las pautas de poblamiento de las regiones, encontrándose índices de movilidad mayores en las regiones de mayor densidad poblacional y donde la población se distribuye en distintos centros urbanos.

Es necesario indagar las características de los desplazamientos de estudiantes en cada una de las zonas de escasa, intermedia y alta movilidad, de modo de entender cómo se comportan en territorios específicos. A continuación se describen las principales características demográficas y territoriales de cada patrón de movilidad y se estudian casos de regiones representativas, tomando como referencia el nivel de educación media en el sector municipal (educación pública). 
Donoso-Díaz y Arias-Rojas | Desplazamiento cotidiano de estudiantes... | OEURE

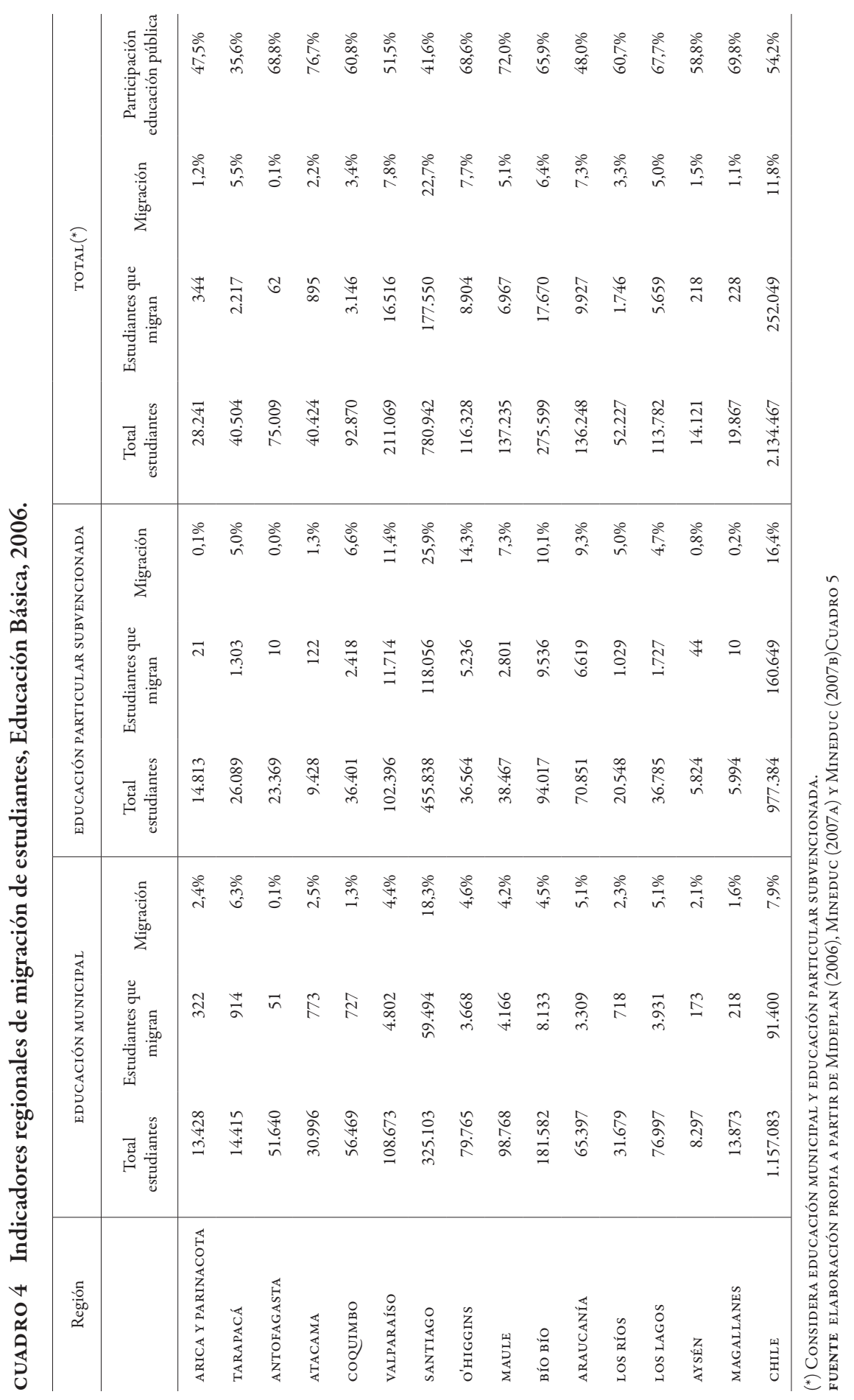




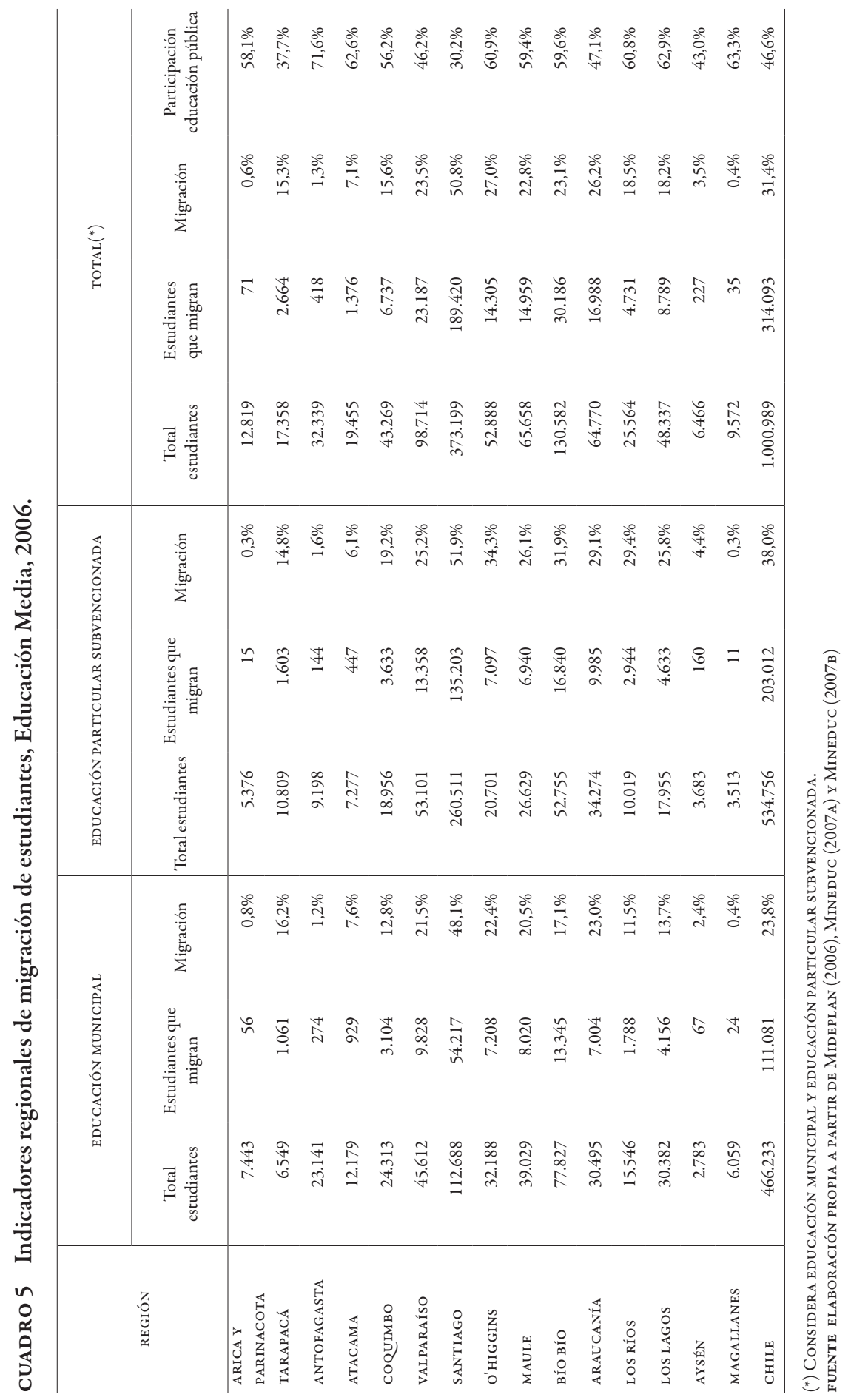




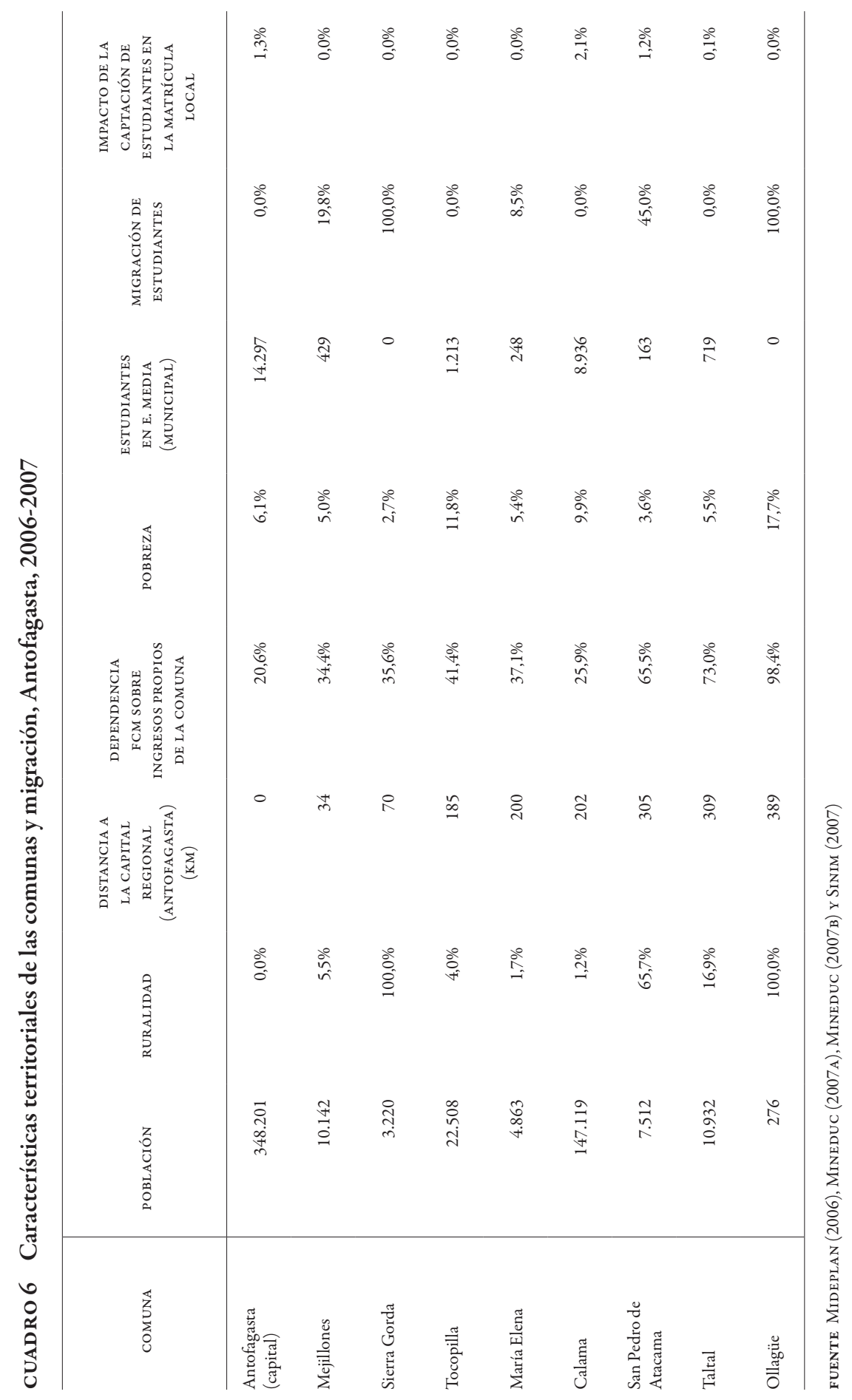




\section{Regiones de escasa movilidad regular}

Las regiones de Arica y Parinacota, Antofagasta, Atacama, Aysén y Magallanes presentan un patrón de baja movilidad regular de estudiantes en educación media municipal, asociado a una población eminentemente urbana, concentrada principalmente en las capitales regionales y provinciales (cfr. Cuadro 3). Si bien los datos con los cuales se trabaja pueden estar sobre o subdimensionando esta situación, lo que se busca es dar cuenta de un fenómeno que requiere atención, más allá de que las cifras finales puedan variar.

En el caso de las regiones mencionadas, los centros urbanos están dispersos en un territorio no solo extenso, sino también de poblamiento relativamente reciente, condiciones agrestes — clima desértico en el norte, estepa y tundra en el sur-, a lo que se suman las diferencias en términos económico-productivos con las demás regiones. Todos ellos son factores que reducen el potencial de desplazamiento cotidiano de los estudiantes allí residentes ${ }^{2}$. En la región de Antofagasta (Figura 1 y Cuadro 6), las comunas de mayor población atraen estudiantes de comunas adyacentes, debido a que algunas no ofrecen educación media municipal (Sierra Gorda, Ollagüe), mientras otras, entregando este tipo de educación, pierden muchos de sus estudiantes. El impacto de este desplazamiento es limitado en las comunas receptoras (principalmente Antofagasta y Calama), debido a las diferencias importantes de magnitud poblacional entre las comunas proveedoras y las receptoras, que disponen de muchos más habitantes que las primeras.

En la región de Aysén (Figura 2 y Cuadro 7) se observa un menor flujo de estudiantes debido a la igualmente menor población de la región, a las mayores distancias entre comunas y a las dificultades de desplazamiento que presenta el territorio, por factores geográficos y climáticos. La mitad de las comunas de la región no tiene oferta de educación media, por lo que los estudiantes deben migrar a otras comunas en forma definitiva, o por temporadas a internados, ya que la movilidad cotidiana como estrategia para acceder a educación secundaria no es posible. En el caso de las comunas que sí actúan como receptoras de estudiantes (Coyhaique, Aysén, Chile Chico), se aprecia un bajo impacto de la captación en la matrícula total de la comuna, que no supera el 6\%, lo cual se explica porque las dos principales comunas de la región (Coyhaique y Aysén) concentran el 80\% de la población regional y, en consecuencia, a la mayoría de los estudiantes del sistema (Donoso, Arias, Cancino \& Oyarzún, 2010).

En las regiones de escasa movilidad diaria, la mayoría de los estudiantes se concentra en pocas comunas que agrupan a la población regional y poseen una importante oferta educativa (educación municipal y educación particular subvencionada, diferentes tipos de enseñanza y diversidad de establecimientos). En tanto, una pro-

2 A estas regiones debe agregarse la región de Tarapacá, a pesar de su mayor nivel de movilidad, por cuanto la mayor frecuencia de este índice se explica por la conurbación que se crea entre la capital regional (Iquique) y una comuna de reciente creación escindida de ella (Alto Hospicio). Por las características de su red educativa y configuración territorial, esta región pertenece al patrón de escasa movilidad regular. 
porción menor se localiza en varias comunas escasamente pobladas, con diferentes grados de ruralidad, las cuales generalmente tienen ofertas educativas limitadas: muchas carecen del nivel de educación media, o bien sus ofertas son solo en ciertas modalidades educativas, con establecimientos que no cubren las demandas (o las expectativas) de los habitantes de la comuna. Es notoria también la ausencia de oferta particular subvencionada en estas comunas. La mayoría de los estudiantes no puede acceder cotidianamente a la oferta de las comunas más pobladas, debido a las distancias y dificultades de acceso. Son, así, más comunes en estas regiones los internados en educación media, la migración definitiva de la población hacia comunas más pobladas, y la deserción escolar o no continuidad en los estudios de los niños y jóvenes, para los cuales la movilidad educativa tiene un costo de oportunidad excesivo.

FIGURA 1 Proporción de estudiantes que migran a otras comunas, educación media municipal, Antofagasta, 2006

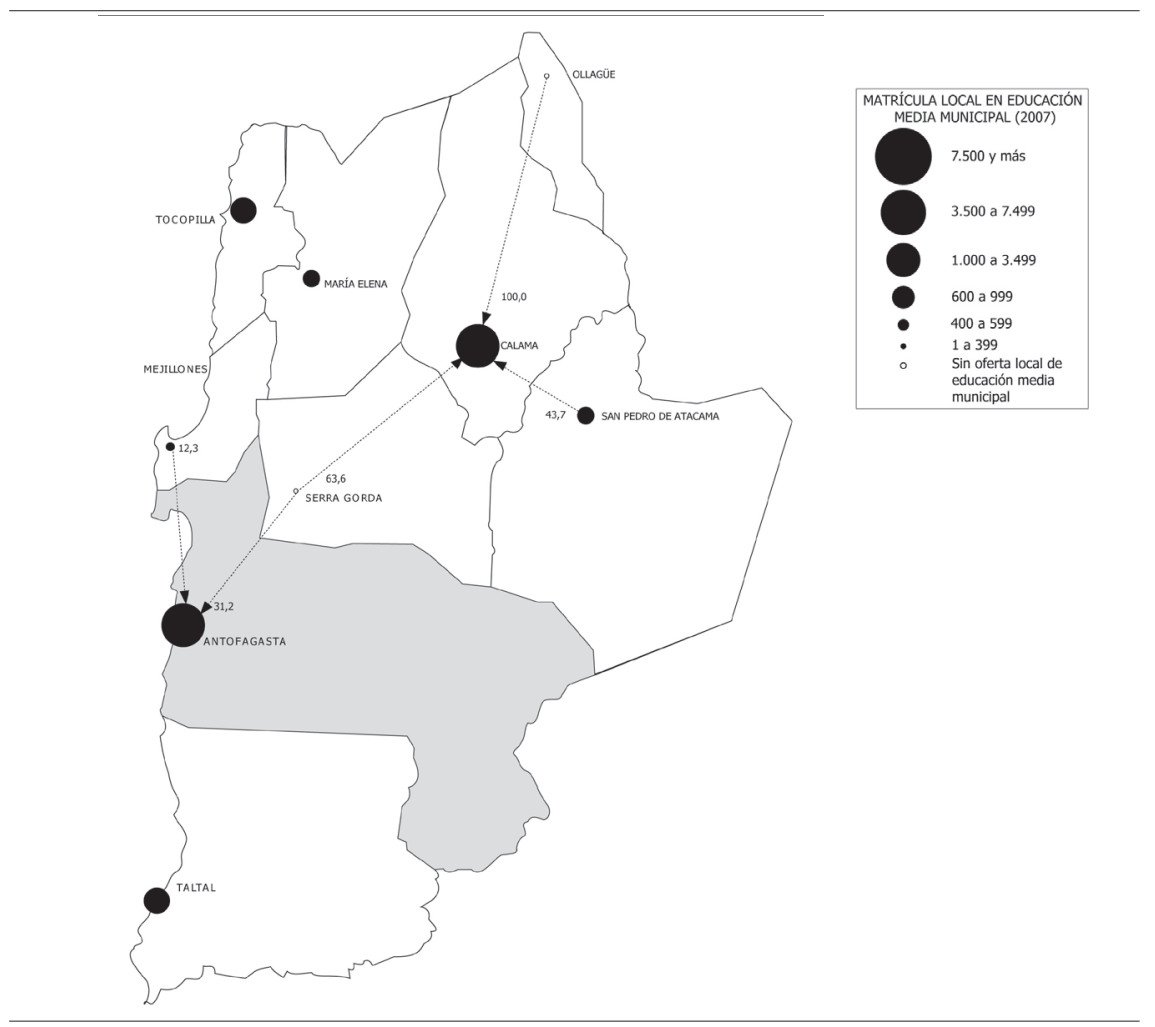

NOTA LOS VALORES REPRESENTAN LA PROPORCIÓN DE PERSONAS QUE VIVEN EN LA COMUNA (NODO DE ORIGEN) Y MIGRAN A LA COMUNA INDICADA CON LA FLECHA (NODO DE DESTINO). SE PRESENTAN LAS RELACIONES MAYORES A $5 \%$ DE LOS ESTUDIANTES LOCALES. EL TAMAÑO DE LOS NODOS ES PROPORCIONAL A LA MATRÍCULA EN EDUCACIÓN MEDIA MUNICIPAL.

FUENTE Elaboración PRopia a PARTir de Mideplan (2006) y Mineduc (2007A y 2007B). 
FIGURA 2 Proporción de estudiantes que migran a otras comunas, educación media municipal, Aysén, 2006

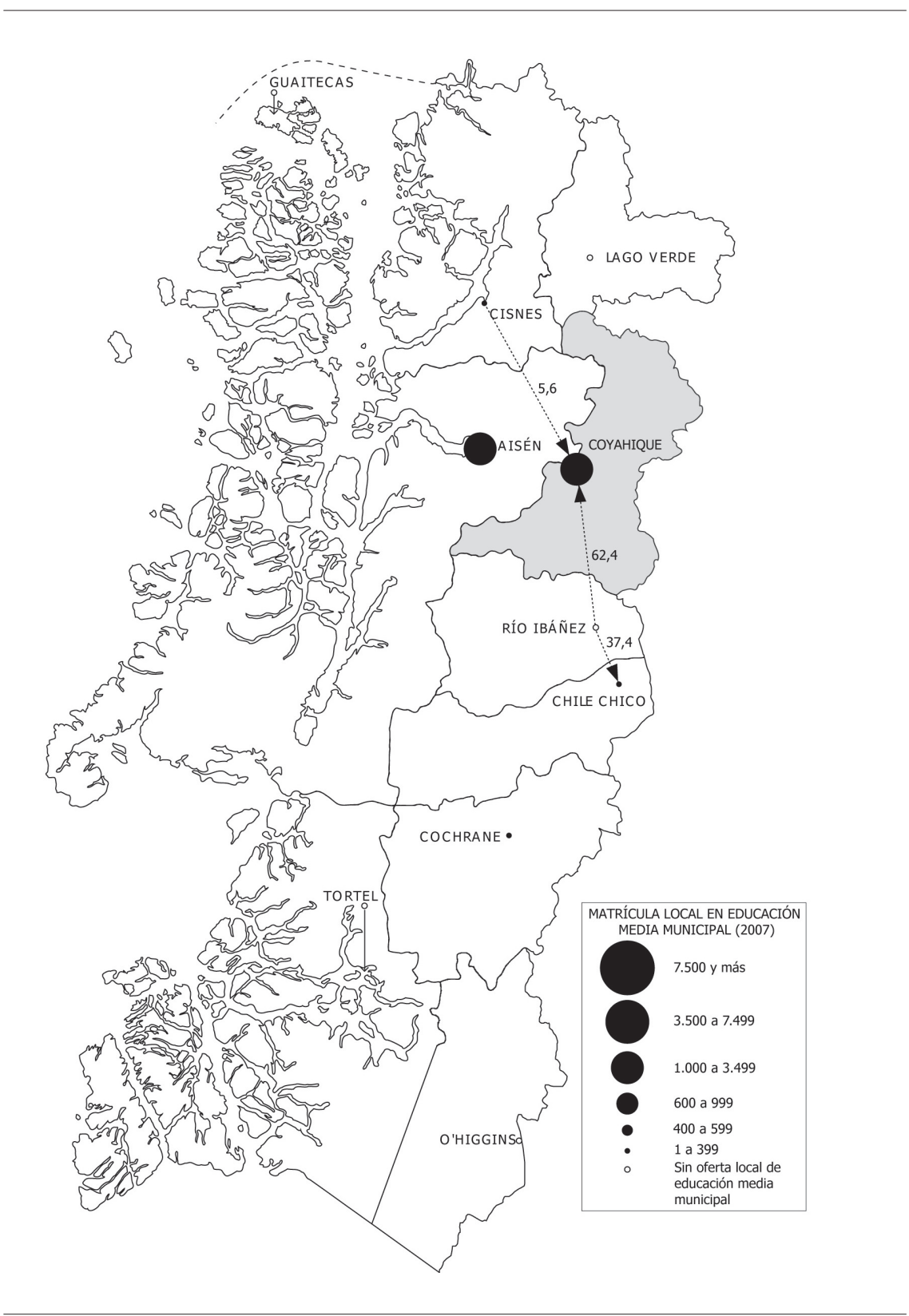

NOTA LOS VALORES REPRESENTAN LA PROPORCIÓN DE PERSONAS QUE VIVEN EN LA COMUNA (NODO DE ORIGEN) Y MIGRAN A LA COMUNA INDICADA CON LA FLECHA (NODO DE DESTINO). SE PRESENTAN LAS RELACIONES MAYORES A MEDIA MUNICIPAL.

FUENTE Elaboración PROPia A partir de Mideplan (2006) y MineduC (2007A y 2007B). 


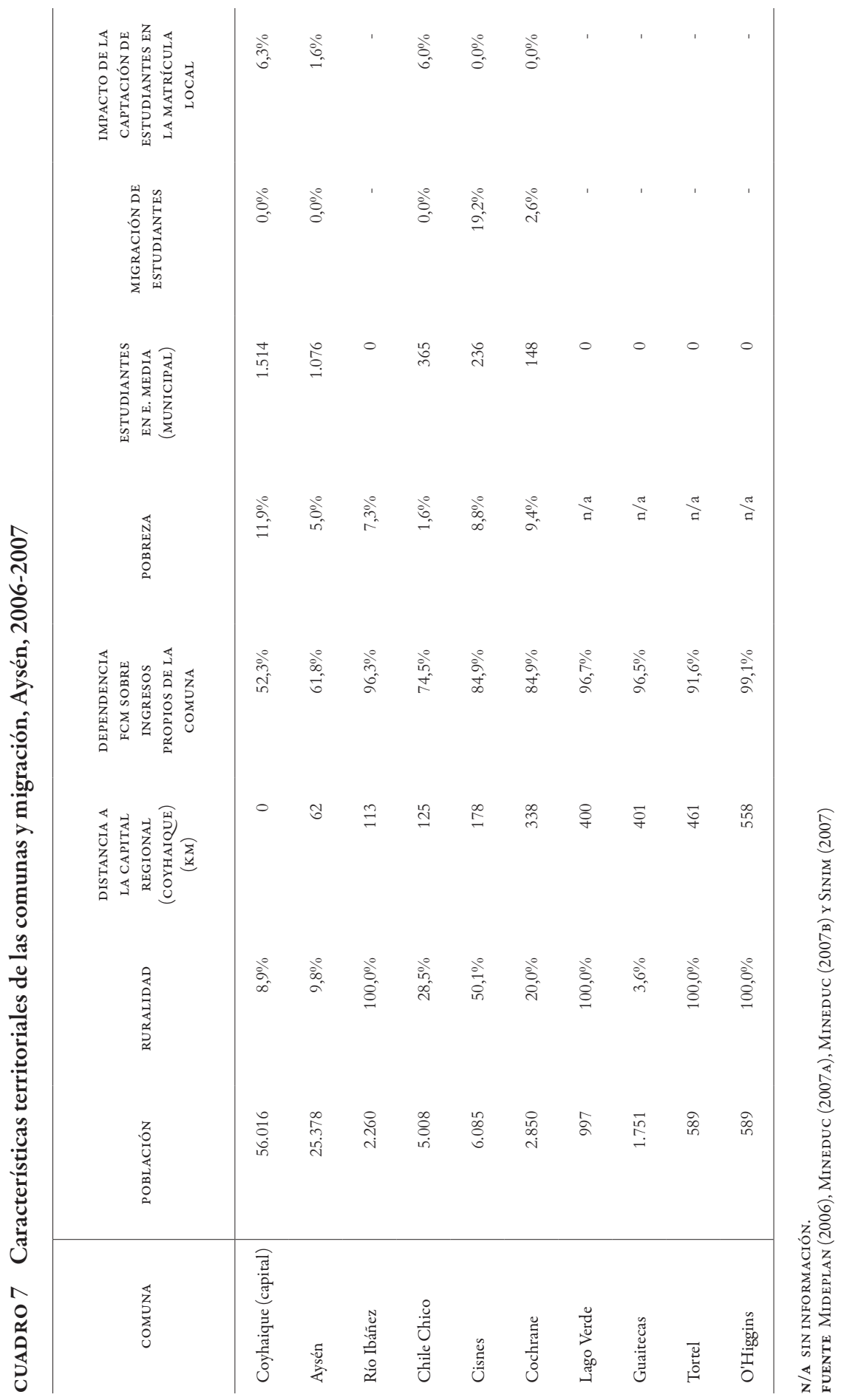




\section{Regiones de movilidad diaria de tipo intermedia}

En las regiones de Coquimbo, Valparaíso, O’Higgins, Maule, Biobío, Araucanía, Los Ríos y Los Lagos, el mayor nivel de movilidad diaria se asocia a una mayor población y a su distribución más homogénea en el territorio (Cuadro 3). Se trata de las regiones donde se concentró tradicionalmente la población del país - de Coquimbo a Biobío, además de los territorios en torno a la ciudad de Valdivia y el archipiélago de Chiloéo bien que recibieron importantes incentivos para su poblamiento: los territorios mapuche entre los ríos Biobío y Toltén y las actuales provincias de Valdivia, Osorno y Llanquihue. La mayor población y su dispersión en el territorio han generado polos urbanos de diversa población, unidos a una importante población rural. Cuando se analizan los flujos de desplazamiento regular de estudiantes en estas regiones, se aprecia la existencia de asociaciones (clusters) naturales de comunas, ligadas por la movilidad de la matrícula; estas asociaciones siguen un patrón de "anillo", en el cual se ubican jerárquicamente en el centro las comunas más importantes de las regiones en términos de población o dinamismo económico - factores determinantes para la diversidad y calidad de la oferta educativa-, con las comunas menos pobladas y de mayor ruralidad y pobreza en la periferia. A su vez, se registran comunas que, situadas sea en la periferia o en posiciones centrales en las regiones, no están imbricadas en los movimientos de estudiantes y quedan fuera de los anillos de desplazamiento identificados.

En la región del Biobío (Figura 3) se establecen diferentes configuraciones en torno a los principales núcleos urbanos de la región. Los Ángeles es el centro de un anillo compuesto por varias comunas de baja población, mientras que Chillán ocupa una posición similar, aunque su cluster incluye una comuna próxima de similar matrícula municipal (San Carlos). La diferencia entre ambos anillos es determinada por la mayor densidad poblacional del territorio en torno a Chillán, comparada con la de Los Ángeles. La estructura de anillo también está presente en la conurbación que forman las comunas del Gran Concepción: a pesar de la mayor población y densidad, se observa el mismo patrón de desplazamiento de estudiantes hacia Concepción, y en menor medida hacia Talcahuano. También se registran relaciones de intercambio de estudiantes entre algunas de las comunas de la provincia de Arauco, pero sin la configuración de anillo en torno a una comuna-foco. Finalmente, de igual manera hay comunas que no se relacionan claramente con un anillo ${ }^{3}$ (Contulmo, Nacimiento y San Fabián), así como otras que forman díadas (Coihueco-Pinto, y Tucapel-Yungay).

El análisis específico del anillo con foco en Los Ángeles (Cuadro 8) muestra dos comunas con muy baja movilidad de estudiantes, Los Ángeles y Mulchén, que también son los territorios con mayor población y mayores sistemas municipales de educación media. A ellas se agrega la comuna de Antuco, de baja población y ubicada en una posición periférica respecto del foco del anillo. Las comunas que menos estudiantes pierden son a su vez las que, igualmente, tienen las poblaciones menos pobres del anillo: mientras que las demás comunas promedian $30 \%$ de pobreza, las de menor movilidad alcanzan el $19 \%$. El promedio de desplazamiento cotidiano de estudiantes de las demás comunas alcanza a $42 \%$. Entre estas comunas de mayor mo-

3 Se consideran como relaciones de flujo de matrícula aquellas superiores al $5 \%$ de los habitantes de la comuna que estudian educación media municipal (esto es, aquellos que estudian en la comuna de origen o fuera de ella). 
vimiento destaca Santa Bárbara, que tiene una de las matrículas de estudiantes más pequeñas, a pesar de ser la tercera comuna en población. Mientras que las comunas proveedoras aportan una proporción importante de sus estudiantes a las receptoras, el impacto de estos estudiantes en las comunas captadoras es bajo: 9\% de la matrícula total en Los Ángeles y $8 \%$ en Mulchén. Por otro lado, es significativo el traspaso de estudiantes que se da en las comunas proveedoras de baja población (como Santa Bárbara, Quilleco y Quilaco), las cuales registran al mismo tiempo baja retención de estudiantes propios y alto impacto de captación de migrantes de otras comunas: sus sistemas educativos son pequeños y, además, atienden a una baja proporción de habitantes de la propia comuna.

FIGURA 3 Proporción de estudiantes que migran a otras comunas, educación media municipal, Biobío, 2006

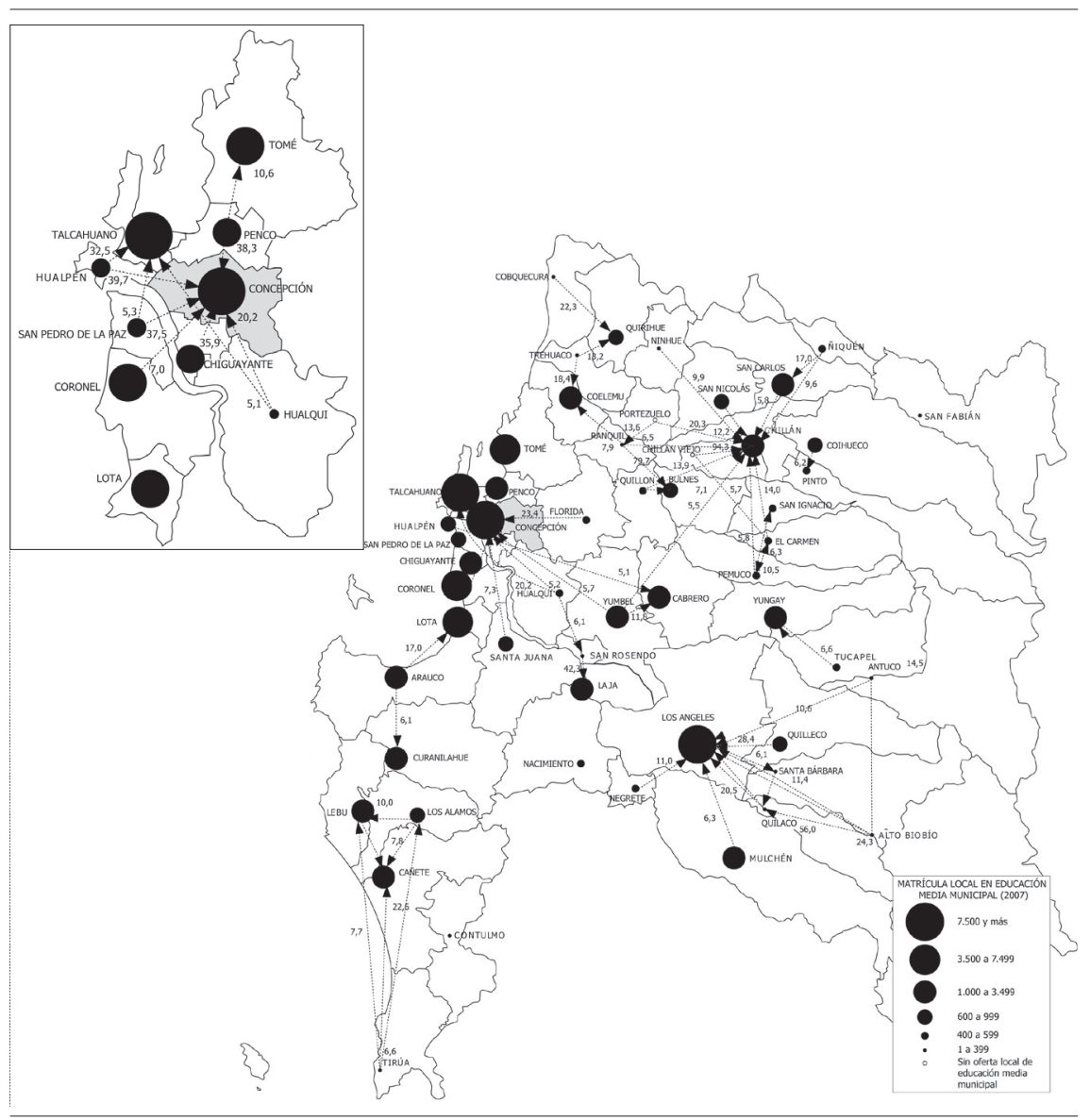

NOTA LOS VALORES REPRESENTAN LA PROPORCIÓN DE PERSONAS QUE VIVEN EN LA COMUNA (NODO DE ORIGEN) Y SE DESPLAZAN A LA COMUNA INDICADA CON LA FLECHA (NODO DE DESTINO). SE PRESENTAN LAS RELACIONES MAYORES A 5\% DE LOS ESTUDIANTES LOCALES. El TAMAÑO DE LOS NODOS ES PROPORCIONAL A LA MATRÍCULA EN EDUCACIÓN MEDIA MUNICIPAL.

FUente elaboración propia a partir de Mideplan (2006) y Mineduc (2007a y 2007B). 


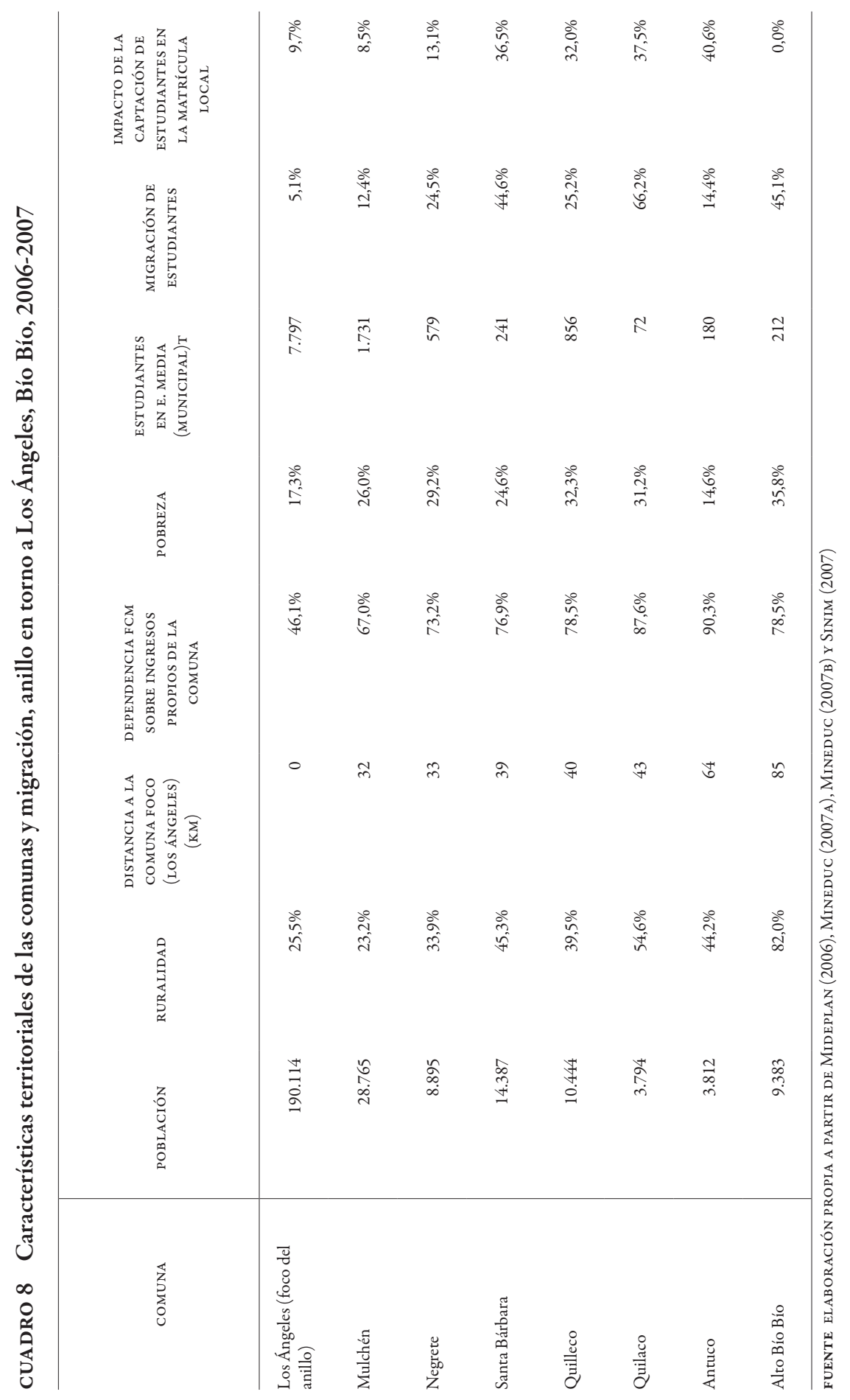


En el caso de la región de la Araucanía, el análisis muestra dos anillos principales, con foco en las comunas de Angol y Temuco, y un anillo más pequeño alrededor de Villarrica (Figura 4). La comuna de Nueva Imperial aparece aislada, y las de Lonquimay y Curacautín forman una díada. Respecto del cluster en torno a Temuco (Cuadro 9), no es clara la misma relación entre pobreza y movilidad de estudiantes que se encontró en el cluster de Los Ángeles. En este anillo parece ser más relevante lo poblado de la comuna y la cercanía a la comuna-foco para explicar el fenómeno del desplazamiento de estudiantes, por cuanto las comunas de menor retención de estudiantes no muestran diferencias importantes en términos de pobreza o dependencia del Fondo Común Municipal (FCM). Más bien, se caracterizan por estar en una posición intermedia entre Temuco y los límites del anillo. Las comunas más lejanas a la capital regional tienden a ser más pobres y, al mismo tiempo, poseen niveles de movilidad inferiores a los de las comunas más ricas próximas al centro de la región. La comuna de Padre Las Casas exhibe un bajo nivel de pobreza y alta población; sin embargo, tiene una baja cantidad de estudiantes y un alto nivel de movilidad, principalmente hacia Temuco, comuna de la que formó parte hasta 1995. La revisión del impacto de los estudiantes captados muestra nuevamente esta relación: a pesar de la alta matrícula del sistema municipal de Temuco, y de su baja movilidad, sobre el $40 \%$ de sus estudiantes proviene de otras comunas, principalmente de Padre Las Casas.

Pese a la importante pérdida de estudiantes de esta última comuna, los estudiantes que capta representan cerca de un tercio de su matrícula. Vilcún y Loncoche son las otras dos comunas del anillo para las cuales la captación de estudiantes es relevante. En la primera de ellas (Vilcún), aunque es una comuna con menor población en situación de pobreza que la segunda, el desplazamiento de estudiantes es alto: casi dos tercios de sus habitantes que estudian educación media municipal lo hacen en establecimientos de Temuco. Como contrapartida, cerca de un tercio de sus estudiantes proviene de otras comunas, como Lautaro y Freire. Por otro lado, la comuna de Toltén muestra una baja movilidad y un alto impacto de la captación de estudiantes en su matrícula (principalmente de Teodoro Schmidt): su posición alejada del centro del anillo, y próxima a la región de Los Ríos (el 7\% de sus estudiantes migra a esta región), podría configurar en el futuro un nuevo anillo, si es que mantiene su nivel de población y aumenta el dinamismo productivo de su territorio y las comunas adyacentes.

Las comunas con bajo impacto de captación de estudiantes (Teodoro Schmidt, Loncoche, Saavedra y Melipeuco) tienen altos niveles de pobreza y están ubicadas a mayor distancia de Temuco. A ellas se agrega Lautaro, que, siendo una comuna aledaña a Temuco, con bajo nivel de pobreza y baja movilidad regular (posee además alta matrícula en educación media municipal), tiene un bajo impacto de captación de estudiantes: es posible que esto se deba precisamente a su cercanía a Temuco, que actúa como una comuna más atractiva para estudiar. Finalmente, se observan tres comunas (Gorbea, Perquenco y Carahue) que se relacionan con el anillo de Temuco pero en forma indirecta, por medio de una comuna intermediaria. 
FIGURA 4 Proporción de estudiantes que migran a otras comunas, educación media municipal, Araucanía, 2006

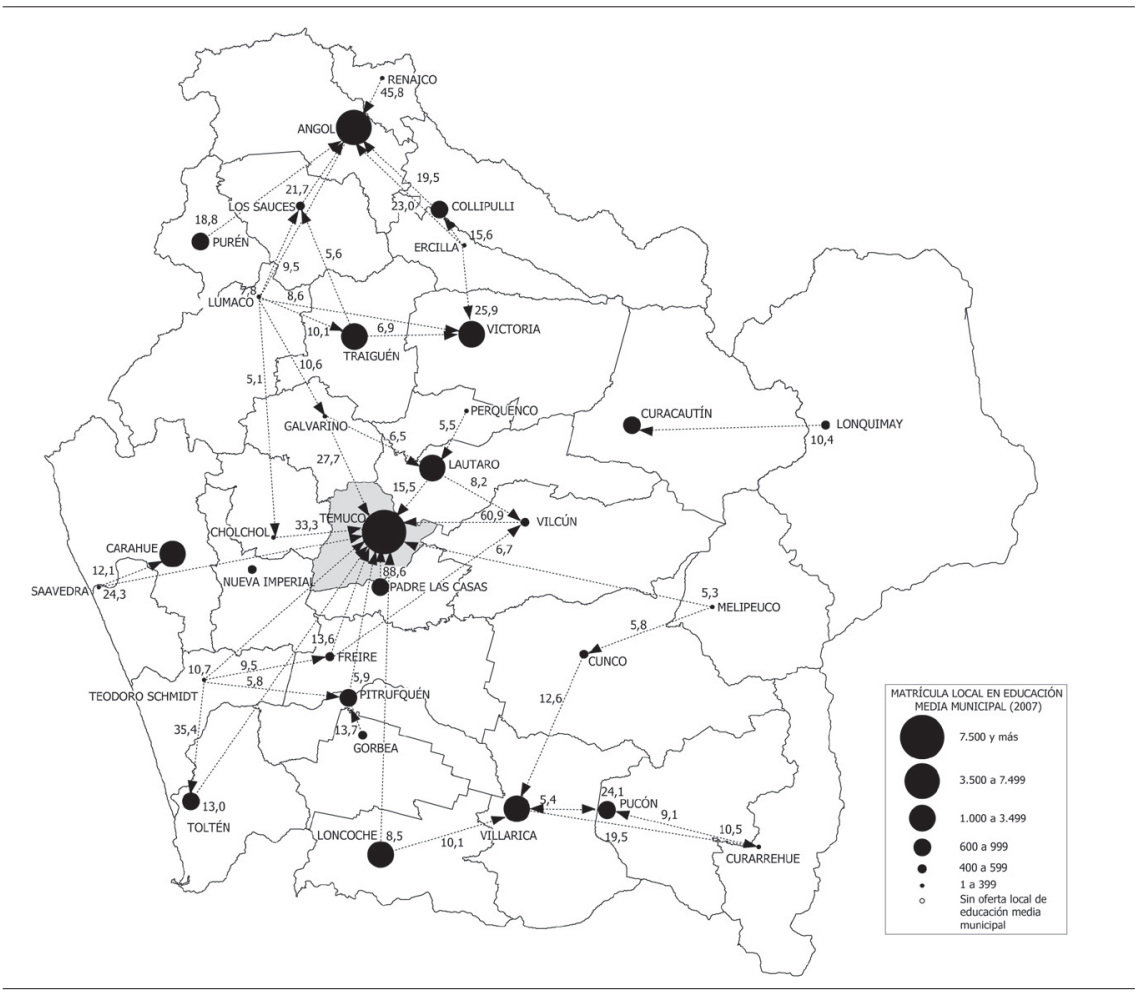

NOTA LOS VALORES REPRESENTAN LA PROPORCIÓN DE PERSONAS QUE VIVEN EN LA COMUNA (NODO DE ORIGEN) Y SE DESPLAZAN A LA COMUNA INDICADA CON LA FLECHA (NODO DE DESTINO). SE PRESENTAN LAS RELACIONES MAYORES A $\%$ DE LOS ESTUDIANTES LOCALES. EL TAMAÑO DE LOS NODOS ES PROPORCIONAL A LA MATRÍCULA EN EDUCACIÓN EDIA MUNICIPAL.

FUENTE ELABORACión PROPIA A PARTIR DE MidePLAN (2006) y MiNeduc (2007A y 2007B).

\section{Regiones de alta movilidad diaria de estudiantes}

La región Metropolitana de Santiago se diferencia de las demás regiones por su elevado nivel de desplazamiento de estudiantes (Cuadros 4 y 5). El análisis de los flujos de matrícula muestra la existencia de dos zonas diferenciadas en el macro territorio (Figura 5): una conformada por las comunas del "Gran Santiago", territorio de alta densidad poblacional, exclusivamente urbano; y otra constituida por comunas suburbanas, que están menos pobladas, presentan población rural —registran en promedio sobre $30 \%$ de ruralidad -4 y concentran cerca del $12 \%$ de la población

4 Para efectos de este estudio, se elaboró una clasificación de comunas, distinguiéndose dos territorios de la región Metropolitana de Santiago: Gran Santiago y Santiago Suburbano. Tenemos así: i) Gran Santiago, con 34 comunas: Cerrillos, Cerro Navia, Conchalí, El Bosque, Estación Central, Huechuraba, Independencia, La Cisterna, La Florida, La Granja, La Pintana, La Reina, Las Condes, Lo Barnechea, Lo Espejo, Lo Prado, Macul, Maipú, Nuñoa, Pedro Aguirre Cerda, Peñalolén, Providencia, Pudahuel, Puente Alto, Quilicura, Quinta Normal, Recoleta, Renca, San Bernardo, San Joaquín, San Miguel, San Ramón, Santiago, Vitacura; y ii) Santiago Suburbano, con 18 comunas: Alhué, Buin, Calera de Tango, Colina, Curacaví, El Monte, Isla de Maipo, Lampa, María Pinto, Melipilla, Padre Hurtado, Paine, Peñaflor, Pirque, San José de Maipo, San Pedro, Talagante, Tiltil. 
de la región (alrededor de 800.000 personas) (Cuadro 10). Es significativo que, a pesar de ello, no alcancen diferencias importantes en sus niveles de pobreza o dependencia del FCM frente al resto de las comunas de la región. La característica de gran desplazamiento de estudiantes de la región Metropolitana se concentra en el primer grupo identificado: comunas del Gran Santiago (Cuadro 11). El grupo de comunas suburbanas posee un comportamiento semejante al observado en regiones de desplazamiento intermedio: si se consideran solo estas comunas, se aprecia que la movilidad comunal promedio de estudiantes alcanza el 19,5\%, muy por debajo del promedio de las comunas del Gran Santiago (53,9\%). Del mismo modo, el impacto de la captación de estudiantes del Santiago Suburbano (7,5\%) es limitado en comparación con el del Gran Santiago (41,9\%). A pesar de las diferencias, los dos grandes sectores de la región no se encuentran separados, existiendo flujos de desplazamiento en las fronteras entre ambos (Figura 5, las comunas de Padre Hurtado, Calera de Tango y Pirque).

FIGURA 5 Movilidad diaria de estudiantes en el Santiago Suburbano y el Gran Santiago, Educación Media Municipal, Región Metropolitana, 2006

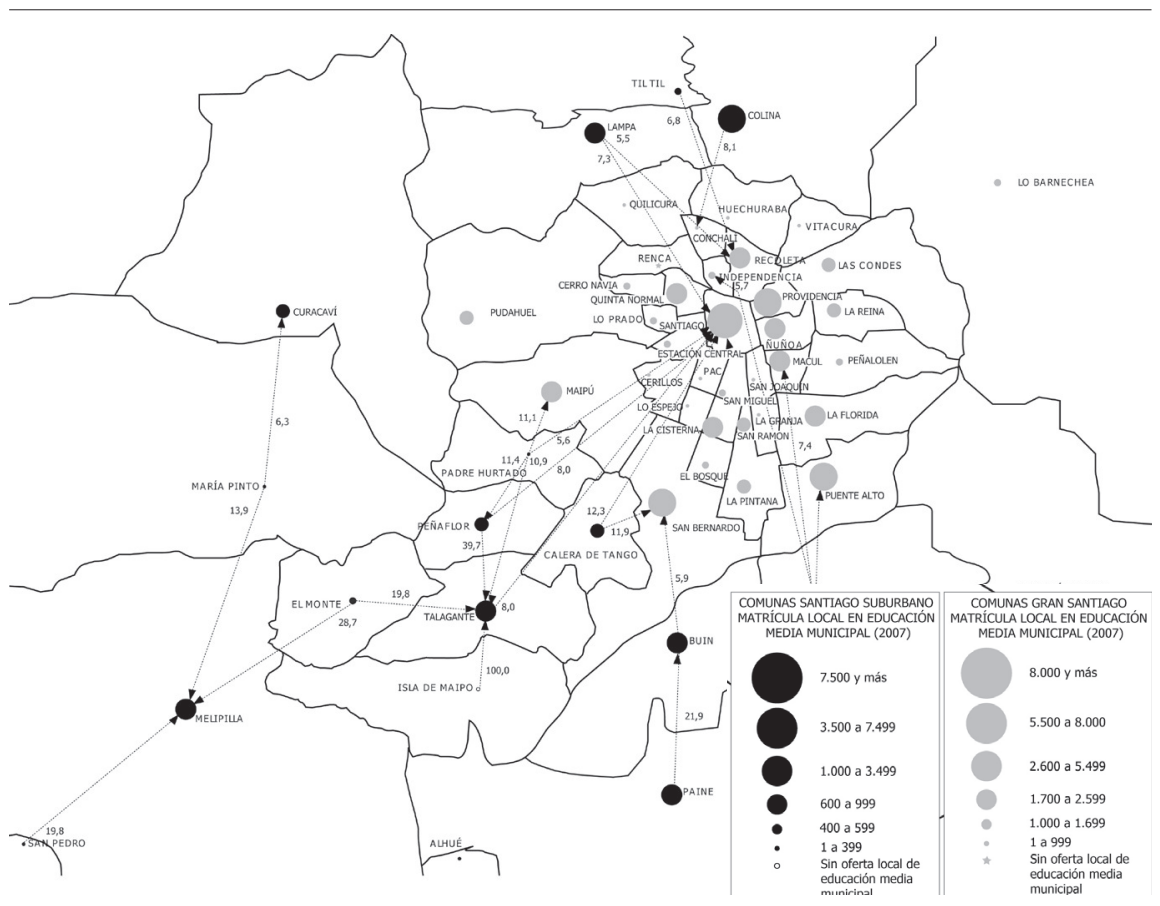

NOTA LOS VALORES REPRESENTAN LA PROPORCIÓN DE PERSONAS QUE VIVEN EN LA COMUNA (NODO DE ORIGEN) Y SE DESPLAZAN A LA COMUNA INDICADA CON LA FLECHA (NODO DE DESTINO). SE PRESENTAN LAS RELACIONES MAYORES A $5 \%$ DE LOS ESTUDIANTES LOCALES. El TAMAÑO DE LOS NODOS ES PROPORCIONAL A LA MATRÍCULA EN EDUCACIÓN MEDIA MUNICIPAL.

FUente Elaboración propia a partir de Mideplan (2006) y Mineduc (2007A y 2007B). 


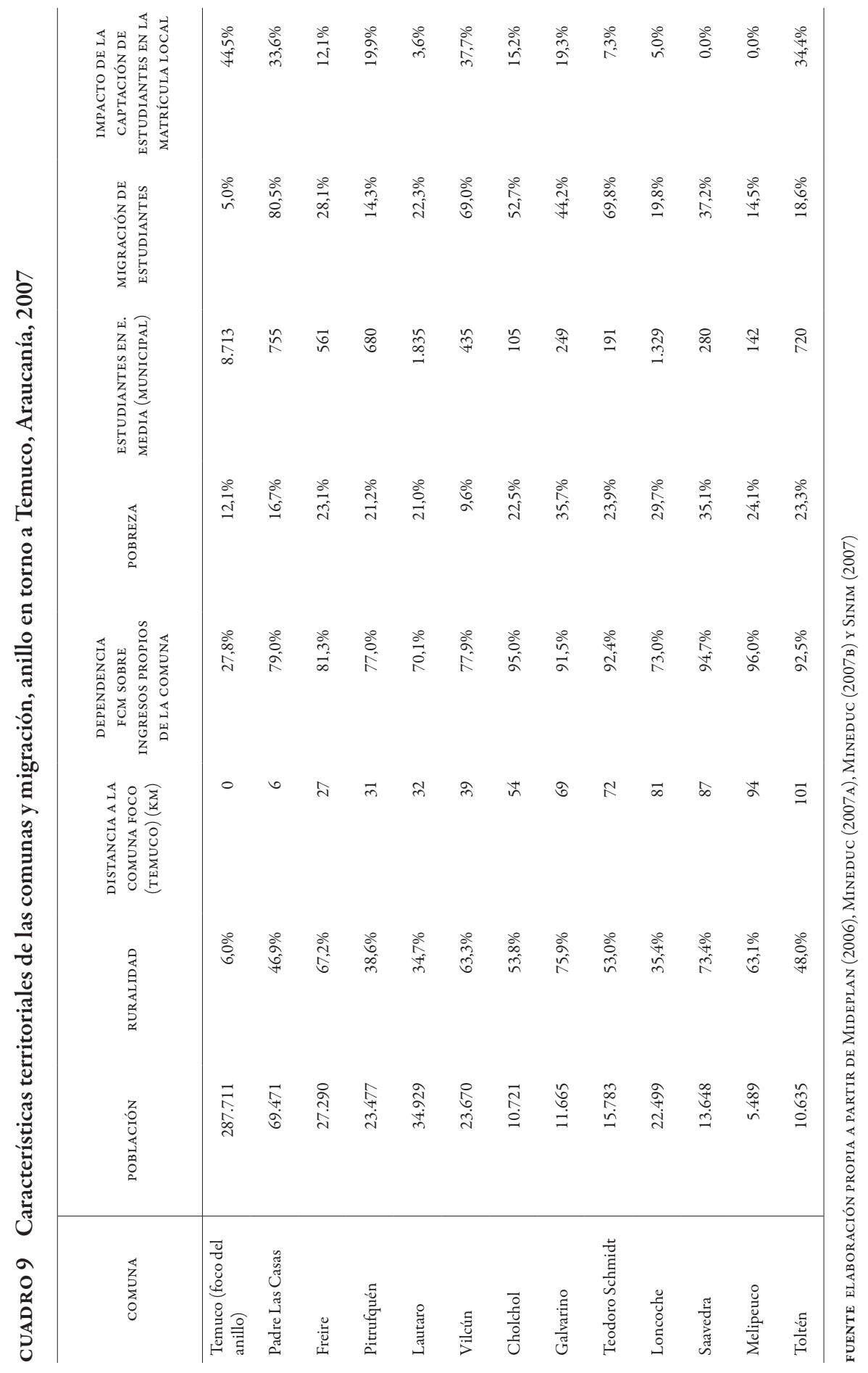


CUADRO 10 Características territoriales de las comunas del Gran Santiago y del Santiago Suburbano, Región Metropolitana de Santiago, 2007

\begin{tabular}{|c|c|c|c|c|c|c|}
\hline & COMUNAS & $\begin{array}{c}\text { POBLACIÓN } \\
\text { TOTAL }\end{array}$ & $\begin{array}{l}\text { POBLACIÓN } \\
\text { PROMEDIO } \\
\text { (COMUNAS) }\end{array}$ & $\begin{array}{l}\text { RURALIDAD } \\
\text { PROMEDIO } \\
\text { (COMUNAS) }\end{array}$ & $\begin{array}{l}\text { DEPENDENCIA } \\
\text { FCM SOBRE } \\
\text { INGRESOS } \\
\text { PROPIOS DE LA } \\
\text { COMUNA }\end{array}$ & POBREZA \\
\hline Gran Santiago & 34 & $\begin{array}{c}5.898 .954 \\
(88,4 \%)\end{array}$ & 173.499 & $0,1 \%$ & $37,4 \%$ & $10,2 \%$ \\
\hline $\begin{array}{l}\text { Santiago } \\
\text { Suburbano }\end{array}$ & 18 & $\begin{array}{l}777.791 \\
(11,6 \%)\end{array}$ & 43.211 & $34,0 \%$ & $47,2 \%$ & $11,7 \%$ \\
\hline $\begin{array}{l}\text { Región } \\
\text { Metropolitana }\end{array}$ & 52 & $\begin{array}{c}6.676 .745 \\
(100,0 \%)\end{array}$ & 128.399 & $11,8 \%$ & $40,8 \%$ & $10,8 \%$ \\
\hline
\end{tabular}

FUENTE Sinim 2007

CUADRO 11 Características de migración educacional de las comunas del Gran Santiago y del Santiago Suburbano, Región Metropolitana de Santiago, 2006

\begin{tabular}{|c|c|c|c|c|c|}
\hline & $\begin{array}{c}\text { Total de } \\
\text { estudiantes }\end{array}$ & $\begin{array}{l}\text { Estudiantes } \\
\text { que migran }\end{array}$ & $\begin{array}{c}\text { Promedio de } \\
\text { estudiantes en E. } \\
\text { Media (comunas) (1) }\end{array}$ & $\begin{array}{l}\text { Retención promedio } \\
\text { de estudiantes } \\
\text { (comunas) (1) }\end{array}$ & $\begin{array}{c}\text { Impacto promedio } \\
\text { de la captación de } \\
\text { estudiantes } \\
(\text { comunas })(1)\end{array}$ \\
\hline Gran Santiago & 96.768 & $\begin{array}{l}52.599 \\
(54,4 \%)\end{array}$ & 2.985 & $46,1 \%$ & $41,9 \%$ \\
\hline $\begin{array}{l}\text { Santiago } \\
\text { Suburbano }\end{array}$ & 20.462 & $\begin{array}{c}3.460 \\
(16,9 \%)\end{array}$ & 1.181 & $80,5 \%$ & $7,5 \%$ \\
\hline $\begin{array}{l}\text { Región } \\
\text { Metropolitana }\end{array}$ & 117.231 & $\begin{array}{c}56.059 \\
(47,8 \%)\end{array}$ & 2.396 & $57,4 \%$ & $30,7 \%$ \\
\hline
\end{tabular}

(1) CONSIDERA A LAS COMUNAS QUE OFRECEN EDUCACIÓN MEDia MUNICIPAL (TODAS CON LA EXCEPCiÓN DE PiRQUE, ISLA DE MAIPO Y RENCA)

FUeNte elaboración propia a partir de Mideplan (2006), Mineduc (2007A), Mineduc (2007b) y Sinim (2007)

El intercambio de estudiantes entre las comunas del Gran Santiago es elevado: alrededor del $50 \%$ de los estudiantes de educación media municipal realizan sus estudios fuera de la comuna donde viven. A diferencia de lo que sucede en las regiones de desplazamiento intermedio, los flujos de estudiantes forman una red densa, de múltiples relaciones entre comunas, sin un foco determinado, pese a la centralidad de las comunas de Santiago y Providencia (Figura 6). Para analizar el comportamiento de la movilidad de estudiantes en esta región fue necesario realizar una caracterización de las comunas del Gran Santiago a partir de las principales 
variables involucradas ${ }^{5}$. Este análisis permitió distinguir cuatro tipologías comunales en el Gran Santiago, además de las comunas de Santiago y Providencia, que se analizaron por separado (Cuadro 12).

FIGURA 6 Proporción de estudiantes que se desplazan regularmente a otras comunas, educación media municipal, región Metropolitana, 2006

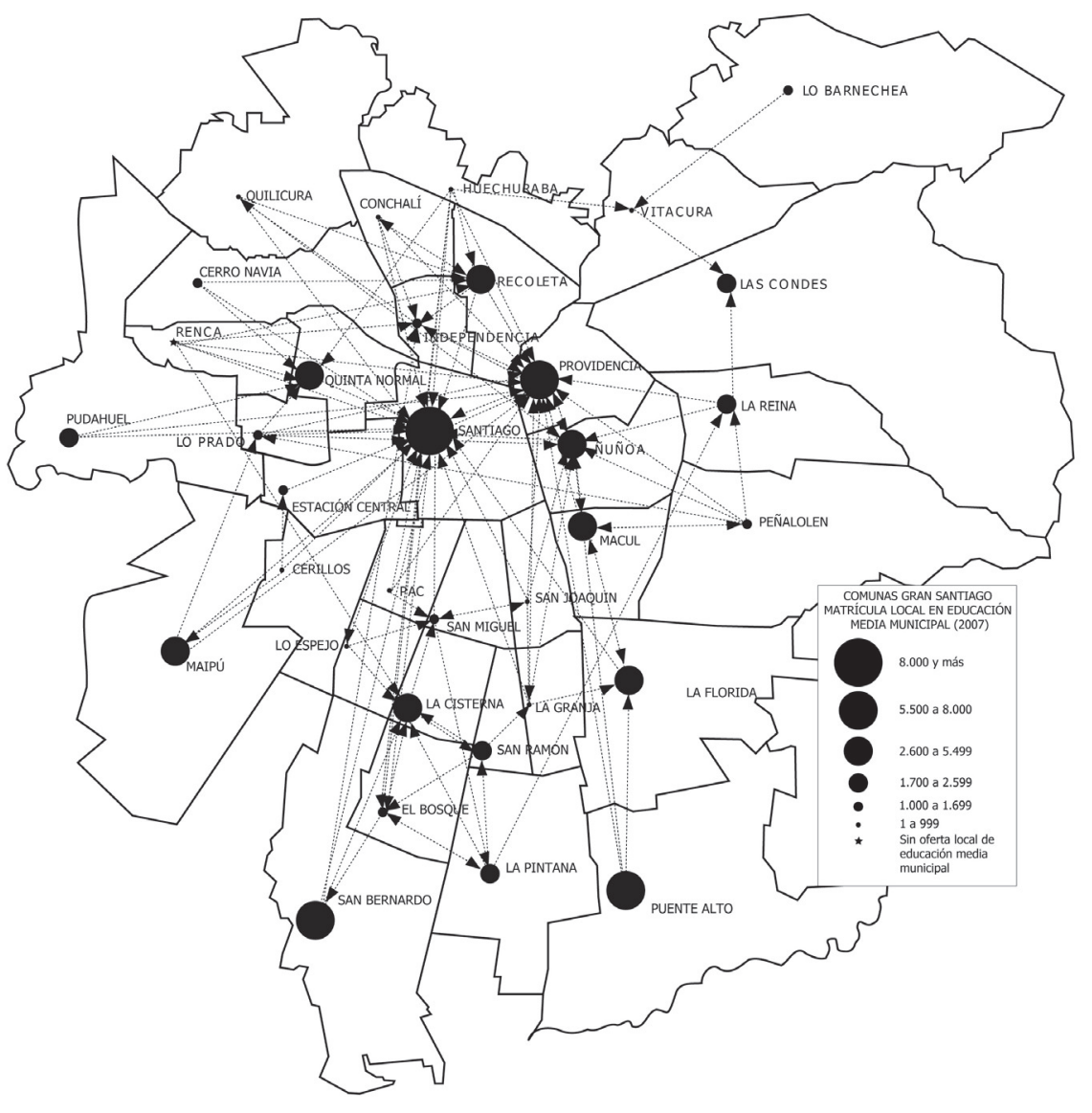

NOTA SE PRESENTAN TODAS LAS RELACiONES MAYORES A 5\% DE LOS ESTUdiANTES LOCALES. El TAMAÑo DE LOS NODOS ES PROPORCIONAL A LA MATRÍCULA EN EDUCACIÓN MEDIA MUNICIPAL.

FUeNTe Elaboración PRopia a PARTir de Mideplan (2006) y Mineduc (2007a y 2007b).

5 La tipología se elaboró a partir del análisis de componentes principales con las variables matrícula, desplazamiento de estudiantes, impacto de la captación de estudiantes en la matrícula municipal, pobreza, participación de la oferta educativa municipal en la comuna y población, identificándose dos dimensiones relevantes. Los puntajes de cada comuna en estas dimensiones se utilizaron para identificar grupos mediante la técnica de clusters de $k$ medias. El comportamiento de las comunas de Santiago y Providencia obligó a excluirlas y analizarlas en forma particular. 
FIGURA 7 Movilidad de estudiantes en el Gran Santiago y tipologías, educación media municipal, región Metropolitana, 2006

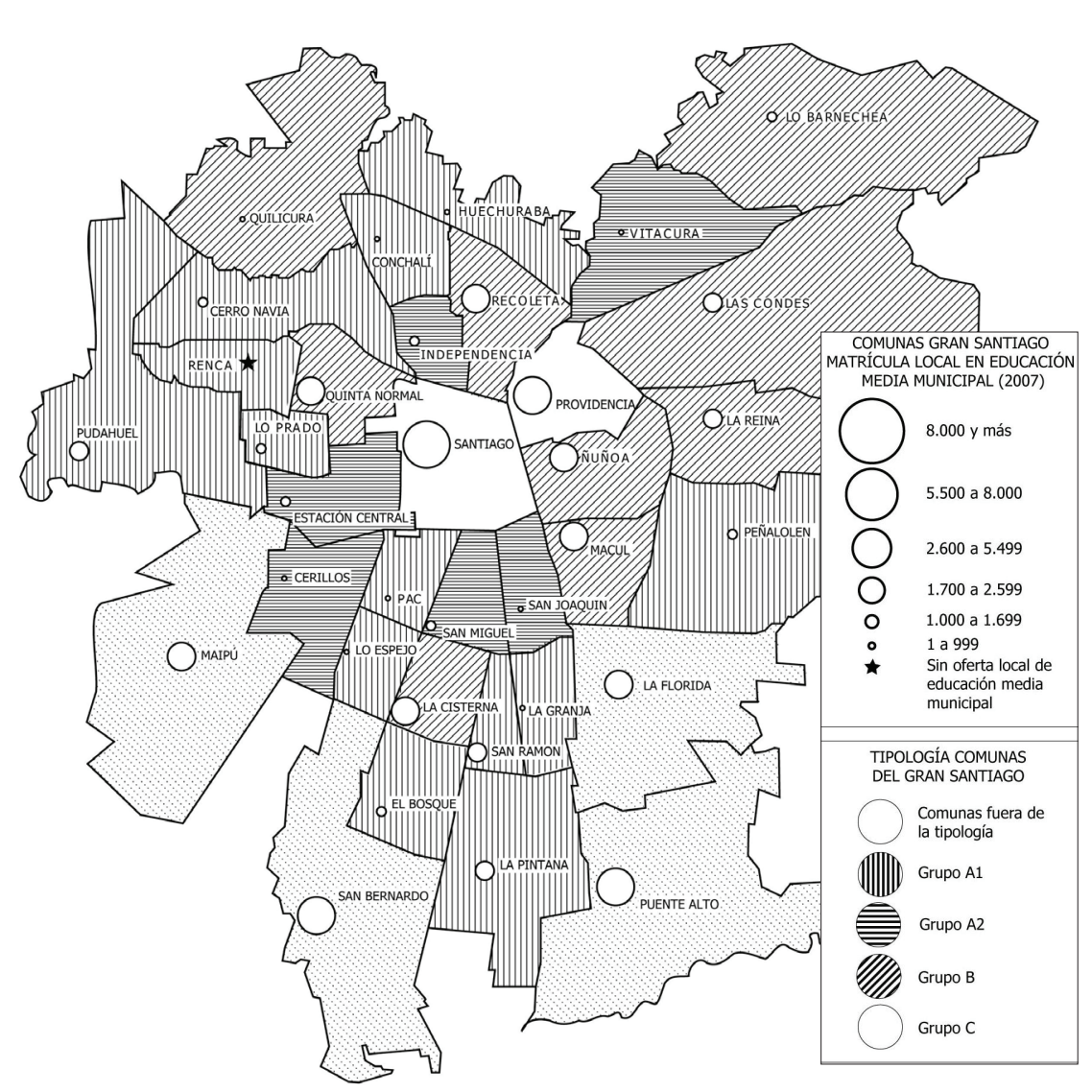

FUente Elaboración propia a partir de Mideplan (2006) y Mineduc (2007a y 2007B).

Las comunas del grupo A1 (Figura 7) presentan una matrícula inferior al promedio de las comunas, y retienen a pocos de sus habitantes en sus propios sistemas municipales de educación. Las comunas del grupo A2, aunque tienen en promedio menor población que el grupo A1, poseen una matrícula similar: esto se debe a que captan estudiantes de otras comunas. Las comunas del grupo B tienen una matrícula en torno al promedio del Gran Santiago. No obstante tener una población similar a las comunas del grupo A1, su pérdida de estudiantes es menor (en torno a un tercio de los estudiantes de la comuna), al tiempo que reciben estudiantes de otras comunas (un poco más del $50 \%$ de sus estudiantes proviene de otras comunas). Finalmente, las comunas del grupo $\mathrm{C}$ presentan los sistemas de mayor tamaño en términos de matrícula. Se trata de comunas masivas ( 500.000 habitantes en promedio), de alta 
movilidad de estudiantes y poco impacto en la captación de estudiantes de otras comunas; no solo son los sistemas de mayor matrícula, sino que también son proveedores importantes para otros sistemas.

CUADRO 12 Tipología de migración educacional comunas del Gran Santiago, Región Metropolitana de Santiago, 2007

\begin{tabular}{|c|c|c|c|c|c|c|c|}
\hline \multirow{2}{*}{ DIMENSIONES } & \multicolumn{4}{|c|}{$\begin{array}{l}\text { TIPOLOGÍA COMUNAS } \\
\text { DEL GRAN SANTIAGO }\end{array}$} & \multicolumn{2}{|c|}{$\begin{array}{c}\text { COMUNAS FUERA DE LA } \\
\text { TIPOLOGÍA }\end{array}$} & \multirow{2}{*}{$\begin{array}{c}\text { PROMEDIO } \\
\left({ }^{*}\right)\end{array}$} \\
\hline & $\operatorname{AI}\left({ }^{* *}\right)$ & $\mathrm{A} 2$ & в & c & SANTIAGO & PROVIDENCIA & \\
\hline Población comunal & 142.893 & 81.019 & 135.044 & 508.857 & 178.818 & 125.584 & 173.499 \\
\hline $\begin{array}{l}\text { Dependencia del FCM } \\
\text { sobre los Ingresos } \\
\text { Propios }\end{array}$ & $62,6 \%$ & $17,5 \%$ & $14,3 \%$ & $55,1 \%$ & $2,8 \%$ & $2,2 \%$ & $37,4 \%$ \\
\hline Pobreza & $13,6 \%$ & $6,0 \%$ & $8,3 \%$ & $12,5 \%$ & $7,3 \%$ & $3,5 \%$ & $10,2 \%$ \\
\hline $\begin{array}{l}\text { Promedio de } \\
\text { estudiantes en E. } \\
\text { Media (Municipal) }\end{array}$ & 1.181 & 1.082 & 2.739 & 5.213 & 23.742 & 7.571 & 2.902 \\
\hline $\begin{array}{l}\text { Migración promedio } \\
\text { de estudiantes }\end{array}$ & $67,5 \%$ & $71,1 \%$ & $35,0 \%$ & $43,8 \%$ & $17,1 \%$ & $60,4 \%$ & $55,1 \%$ \\
\hline $\begin{array}{l}\text { Impacto promedio } \\
\text { de la captación de } \\
\text { estudiantes }\end{array}$ & $20,9 \%$ & $69,2 \%$ & $51,4 \%$ & $14,1 \%$ & $89,6 \%$ & $88,9 \%$ & $40,7 \%$ \\
\hline Comunas & 13 & 6 & 9 & 4 & 1 & 1 & 34 \\
\hline
\end{tabular}

$(*)$ Todas las comunas del Gran Santiago.

(**) InCluye a La COMUNA de RenCA, QUE eS LA ÚNICA QUE El AÑo 2006 No PRESENTA EDCUACION MEDIA MUNICIPAL EN EL GRAN SAN

fuente elaboración propia a partir de Mideplan (2006), Mineduc (2007A), Mineduc (2007 B) y Sinim (2007)

La diferencia entre comunas proveedoras o captadoras aparentemente está relacionada con las condiciones económicas de las mismas: las comunas del grupo A2 promedian menos de la mitad de la proporción de pobreza que las comunas del grupo A1, y sus presupuestos municipales dependen mucho menos del FCM (17\% contra 62\%). Además, es notorio que las comunas del grupo A1 se ubican en zonas más distantes al centro de la ciudad que las del grupo A2 (Figura 7): zonas periféricas, de poblamiento más reciente, constituidas fundamentalmente por viviendas sociales o de bajo costo, con equipamiento urbano deficitario y concentración de hogares de bajos ingresos. También se establece una diferencia en las condiciones 
económicas cuando se comparan los grupos B y C. Las comunas del grupo B, al mismo tiempo que retienen y captan más estudiantes que las del grupo $\mathrm{C}$, presentan menores niveles de pobreza de su población y menor dependencia del FCM.

Las comunas de Santiago y Providencia se trataron como casos diferentes, ya

que sus niveles de matrícula y de intercambio de estudiantes son muy diferentes de los del resto. Mientras que la suma de ambas comunas representa cerca del 5\% de la población del Gran Santiago, solo en la comuna de Santiago asiste el 24\% de la matrícula municipal de educación media, en tanto que en Providencia estudia el $7,8 \%$. Esto significa que gran parte de sus estudiantes proviene de otras comunas: en efecto, casi el 90\% de los estudiantes de ambas comunas está en esta condición (Cuadro 12). A pesar de estas diferencias, existe semejanza con los grupos de comunas descritos: se verifica nuevamente la asociación entre captación de estudiantes y nivel socioeconómico de la comuna: tanto en la comuna de Santiago como en la de Providencia, los niveles de pobreza son menores que el promedio del Gran Santiago, y su dependencia del FCM es mínima.

\section{Discusión}

El desplazamiento cotidiano o movilidad regular intercomunal de estudiantes en el sistema escolar chileno es un fenómeno insuficientemente estudiado en su magnitud e impacto. El análisis de esta problemática muestra que se dan comportamientos diferenciales según la dinámica de poblamiento y desarrollo del territorio, que genera formas específicas de vinculación entre comunas, por medio de la movilidad de estudiantes. Sin embargo, pese a sus impactos en el marco organizacional, normativo y financiero de la educación pública chilena, tales variaciones no están consideradas en su institucionalidad actual. Precisamente, debido a que la institucionalidad educativa actual otorga escasa relevancia a la dimensión territorial, en la discusión acerca de las nuevas formas de institucionalidad de la educación pública se asume el territorio como un dato de proximidad, para efectos de una gestión más eficiente; pero ello se hace sin analizar cuáles son los territorios ya configurados por las dinámicas de desplazamiento de la población de estudiantes, que reflejan el racional bajo el cual se estructuran los anillos de relaciones. Es decir, existe una arquitectura territorial de hecho en este plano, que debiese ser reconocida por la nueva propuesta que se elabore en esta materia, de forma que, partiendo de tal situación, aumenten las probabilidades de éxito al respecto.

En segunda instancia, la situación descrita implica diseñar un marco normativo diferente de la identidad comuna/territorio que se da mecánicamente en la actualidad. Siendo evidente que los anillos de relaciones conforman nuevos territorios que incluyen comunas o parte de ellas, la gestión y las normas al respecto deben cambiar y, por ende, también deben hacerlo las entidades responsables de su dirección.

En tercer lugar, esta realidad implica revisar el sistema de financiamiento de la educación pública y privada vía subsidio, pues en su formato actual permite que las relaciones de desplazamiento entre comunas se vinculen con la desigualdad en la 
distribución territorial del conjunto de oportunidades de distinto tipo — productivas, económicas, culturales, entre otras- existente en el país. Este proceso, que a su vez ocurre en el contexto de una creciente segmentación educativa (Cuadro 1), debería ser considerado por el sistema de financiamiento de la educación pública y subvencionada, debido al alto impacto que posee sobre ambos sistemas (Roemer, 1998). La relevancia del tema, así como su magnitud, muestran que la incorporación de la dimensión territorial en las propuestas de perfeccionamiento de la institucionalidad educativa es un factor clave para superar las restricciones actuales del diseño, cuya ausencia aumenta de modo considerable el riesgo de fracaso.

Los resultados muestran, específicamente, que la movilidad: i) es mayor en educación media en comparación con educación básica; y ii) es mayor en la educación particular subvencionada que en la educación municipal (pública). La revisión de la movilidad regular de estudiantes de educación media municipal a nivel regional dio cuenta de tres zonas diferenciadas, coincidiendo los niveles de movilidad con los patrones característicos de poblamiento regional: regiones extremas de población reducida y concentrada (escasos desplazamiento o movilidad), regiones centrales de mayor población y ruralidad (intermedia), y una región metropolitana muy poblada y de gran densidad (alta movilidad).

En las zonas extremas se estableció que las redes de desplazamiento son escasas, debido tanto a las distancias entre los centros poblados como a la concentración de la población en pocos núcleos urbanos y a la reducida población de estudiantes, además de factores climáticos severos en algunas de ellas. Las regiones centrales, en cambio, debido a su mayor población y a la dispersión de esta en el territorio, forman asociaciones naturales (clusters) de comunas. Estos aspectos son de relevancia para la discusión acerca de las nuevas estructuras institucionales de la educación pública, atendiendo a la dimensión territorial y al desplazamiento regular de estudiantes como factor clave para su desarrollo.

Se observa que las comunas menos pobres tienden a atraer a estudiantes de zonas de mayor pobreza y precariedad, que suelen ubicarse en zonas periféricas de los anillos. Los sistemas públicos de estas comunas periféricas son los que pierden más de sus estudiantes a favor de sistemas de otras comunas, a menos que se encuentren en territorios aislados (anillo de Temuco). Por otro lado, son interesantes dos fenómenos: i) que las relaciones de desplazamiento tienden a una direccionalidad desde comunas menos pobladas y desarrolladas hacia comunas más dinámicas de mayor población —no se aprecian, por ejemplo, muchas relaciones de intercambio de estudiantes en dirección opuesta a la señalada o entre comunas semejantes-; y ii) que existen sistemas de educación municipal "deslocalizados": sistemas cuyos habitantes, en proporción importante, se desplazan a otras comunas más centrales y de mayor población (alta movilidad), pero que a su vez nutren su propio sistema con estudiantes de otras comunas más periféricas y de menos habitantes (alto impacto de la captación de estudiantes), como es el caso de Santa Bárbara (en el anillo de Los Ángeles, Figura 3) y Vilcún (en el anillo de Temuco, Figura 4). 
En todas las regiones centrales de movilidad intermedia se establece un racional similar de asociación: anillos de comunas escasa o moderadamente pobladas ("proveedoras") asociadas a comunas de mayor desarrollo poblacional y productivo ("receptoras"), que generalmente corresponden a capitales regionales o provinciales. A pesar de que las comunas proveedoras aportan una proporción importante de sus habitantes a los sistemas de las comunas receptoras, el impacto directo de este intercambio es menor para las comunas receptoras, debido a las importantes diferencias en los tamaños brutos de población. Sin embargo, a pesar de que el impacto directo sea bajo, hay efectos secundarios: de acuerdo con la tendencia que asocia los desplazamientos con mayores ingresos, puede inferirse que quienes estudian en comunas receptoras tienden a tener mejores niveles de educabilidad. Asimismo, estos estudiantes probablemente tenderán a desplazarse en forma permanente a la comuna receptora, haciendo un aporte a su capital humano. En este sentido, además de los impactos directos en las matrículas de las comunas receptoras y proveedoras, deben considerarse los impactos indirectos de este tipo de desplazamiento, que pueden manifestarse en migraciones permanentes de población y en la distribución del capital humano en las comunas, indispensable para un desarrollo más equitativo del territorio. Por otro lado, el fenómeno de la movilidad — desde la perspectiva de las comunas receptoras-, aunque de bajo impacto, permite que algunos sistemas comunales de educación pública reemplacen parte de los estudiantes que pierden por la competencia de los establecimientos particulares subvencionados, fenómeno creciente en el último tiempo respecto de la tendencia de los últimos veinticinco años, especialmente en los sectores de ingreso medio.

Las redes de relaciones entre comunas proveedoras y receptoras muestran que los anillos se superponen a territorios de desarrollo desigual. Los flujos de desplazamiento diario reflejan esta desigualdad, la que se asocia no solo con el nivel de poblamiento, el desarrollo productivo de las comunas, la condición de pobreza de sus hogares o la educabilidad compleja de sus estudiantes. Más bien, tiene que ver con una institucionalidad de la educación pública que no se hace cargo de las diferencias derivadas del origen social - y territorial, en este caso-. De este modo, el sistema educativo es incapaz de asegurar sin discriminaciones el derecho a la educación, aspecto clave en la política pública de educación y determinante en el cumplimiento de uno de los roles clave que le competen en la sociedad. Solo en este contexto se explica que los habitantes de comunas periféricas rechacen aquellos establecimientos que se ubican más próximos a sus hogares, y destinen tiempo y recursos para desplazamientos cotidianos a comunas lejanas en busca de un establecimiento que satisfaga en mayor medida sus expectativas educacionales. En este sentido, no solo es relevante indagar en el futuro respecto de qué es lo que estos estudiantes y sus hogares valoran de los establecimientos de comunas receptoras, sino también respecto de qué los diferencia de quienes no se desplazan, en términos de racionales de elección, condiciones materiales y trayectorias educativas y laborales futuras.

En razón de ello, la identificación de agrupaciones o clusters "naturales" da cuenta de un comportamiento de la población que obedece a patrones racionales y que 
propone estructuras de asociación que deben significar, en términos de la discusión de propuestas en esta materia, criterios relevantes de considerar cuando se concilian los desafíos de la educación pública y los problemas de escala que presenta en algunos territorios, los mismos enfoques que deben reflejarse en forma eficiente en los nuevos instrumentos de financiamiento que se propongan. Adicionalmente, estas nuevas estructuras han de implicar un fortalecimiento para todos los actores institucionales, comunas proveedoras y receptoras, y no solamente para las receptoras. Esto significa generar políticas e instrumentos que estimulen y a su vez garanticen estos aspectos, y además potencien la asociatividad, con un sentido territorial.

La región Metropolitana de Santiago mostró dos realidades distintas: las comunas suburbanas - que componen un territorio de comportamiento similar a las regiones de desplazamiento intermedio-, y las comunas del Gran Santiago. El conjunto de estas últimas da cuenta de altos niveles de movilidad: en torno a $50 \%$ de los estudiantes asiste a un establecimiento fuera de su comuna de origen, lo que se materializa en múltiples relaciones de intercambio de estudiantes entre comunas. Sin embargo, la red de relaciones de traslado de estudiantes, a pesar de ser más densa, sigue el mismo patrón de las regiones intermedias: los desplazamientos tienen una direccionalidad desde sistemas públicos de comunas de mayor pobreza, más periféricos y estigmatizados, hacia comunas menos marginales.

El estudio de tipologías de comunas del Gran Santiago (Donoso \& Arias, 2011) mostró además que, mientras la mayoría de las comunas puede ser clasificada de acuerdo con su nivel de pobreza, características de desplazamiento de estudiantes y matrícula, las comunas de Santiago y Providencia constituyen categorías de excepción. Y ello principalmente porque los estudiantes que asisten a sus establecimientos de educación media municipal superan con mucho la demanda de sus propios habitantes: se trata de dos comunas que no solo ocupan una posición central en la ciudad, sino que al mismo tiempo heredaron, luego del proceso de municipalización de los años ochenta, una buena parte de la inversión pública en educación de Santiago. Tal circunstancia les permite contar con establecimientos públicos prestigiosos, con una cantidad de vacantes que no dice relación con su población local, y además ser selectivas en el acceso de estudiantes. Por otro lado, comunas de gran población (grupo C: Puente Alto, Maipú, La Florida, San Bernardo) tienen sistemas municipales proporcionalmente pequeños: Maipú, con 700.000 habitantes en 2006 tenía una matrícula en educación media municipal similar a Quinta Normal, comuna de 90.000 habitantes.

Las dinámicas de crecimiento de la ciudad de Santiago en los últimos años, unidas a la política de municipalización de la educación pública iniciada hace treinta años (Paredes, 2010), han implicado una concentración de la inversión en infraestructura pública en algunos sectores de la ciudad, en desmedro de otros: la comuna de Santiago, principalmente, luego de la municipalización, recibió una parte importante de la inversión fiscal de los años anteriores en infraestructura en educación media, ampliándose así su oferta potencial. El proceso inverso tuvo lugar a partir 
de mediados de los noventa, cuando tuvo lugar un crecimiento importante de la población en comunas periféricas de la capital (un ejemplo: Maipú pasó de 256.000 habitantes en 1992, a casi 700.000 en 2007): estas se enfrentan a una demanda educativa que su sistema municipal no puede satisfacer.

Con distintos matices, asociados a sus características propias, el fenómeno de la movilidad cotidiana de estudiantes en las distintas regiones da cuenta de que la institucionalidad pública implementada desde los años ochenta muestra dificultades para entregar una oferta educativa de calidad que permita equilibrar en alguna medida las diferencias de origen de los estudiantes. Y no solo eso, sino que la institucionalidad vigente también opera ocultando los desafíos que imponen el territorio y sus desiguales condiciones de desarrollo, al logro de esa meta. En este sentido, el diseño municipalizado determina estructuras de gestión y regulación a escala local, pero no en niveles agregados. El estudio ha mostrado que a nivel agregado se desarrollan fenómenos de movilidad de estudiantes asociados a oportunidades educativas diferenciadas y, por lo mismo, vinculados al desarrollo territorial. Actualmente tales circunstancias constituyen espacios de discriminación tanto para los habitantes de zonas rurales o núcleos urbanos pequeños, como para los habitantes de zonas extremas y hogares ubicados en la periferia de la ciudad de Santiago, aspectos que actualmente el sistema educacional no considera, y menos compensa.

En este sentido, es de relevancia en el debate actual respecto del perfeccionamiento de la institucionalidad de la educación pública, incorporar la dimensión territorial en las propuestas de política, especialmente en un contexto de fuerte competencia con el sector particular subvencionado. El aumento en la sinergia en los sistemas locales y entre estos mismos subsistemas podría hacer más eficientes sus márgenes de operación y, lo más importante, les permitiría cumplir con el deber constitucional de garantizar a los ciudadanos una educación de calidad para todos, especialmente en las regiones de movilidad intermedia y baja. En las primeras, por cuanto concentran cerca del $60 \%$ de los estudiantes de la educación pública y en ellas la educación particular ha tenido una penetración menor, debido a los mayores niveles de pobreza de sus habitantes y a los costos asociados a la ruralidad. En este contexto, el desarrollo coordinado de los clusters educativos podría generar una oferta pública de mejor calidad, con mayor estatus y, por lo tanto, más atractiva y validada para la población escolar. Las segundas - aquellas regiones de escaso desplazamiento, con una oferta de educación esencialmente pública- requieren de procesos asociativos con fuerte soporte del Estado, sea del subnacional o del nacional, para enfrentar este desafío, y en ellas el aumento en la sinergia de los sistemas locales permitiría compensar en parte las inequidades territoriales.

Un caso aparte lo constituye el Gran Santiago. La Figura 8 muestra cómo se relacionan la matrícula de los sistemas públicos locales de educación con los habitantes de las comunas que efectivamente asisten a este tipo de educación (en cualquier comuna): se aprecia con claridad la penetración de la educación privada, especialmente en las comunas más pobladas. Al menos en el caso del Gran Santiago, el hecho de que muchos agentes privados estén dispuestos a abrir escuelas (la subvención del Estado - incluyendo la subvención preferencial-y las pocas regulaciones son un 
gran incentivo) y, además, el que el desarrollo de la educación pública sea riesgoso para las comunas (se debe operar con regulaciones menos flexibles, con estudiantes más costosos de educar, y los recursos para invertir — cuando los hay- en nuevas escuelas y más profesores tienen costos de oportunidad importantes), podría incidir en la pasividad de algunas comunas para enfrentar el problema (Valenzuela, 2006). En este sentido, para muchos de los encargados de la administración de la educación pública, la "competencia" del sector particular subvencionado es más bien una "colaboración". oEURE

FIGURA 8 Matrícula de los sistemas locales de educación pública, habitantes que asisten a establecimientos públicos y tipologías comunales, educación media municipal, Gran Santiago, 2006

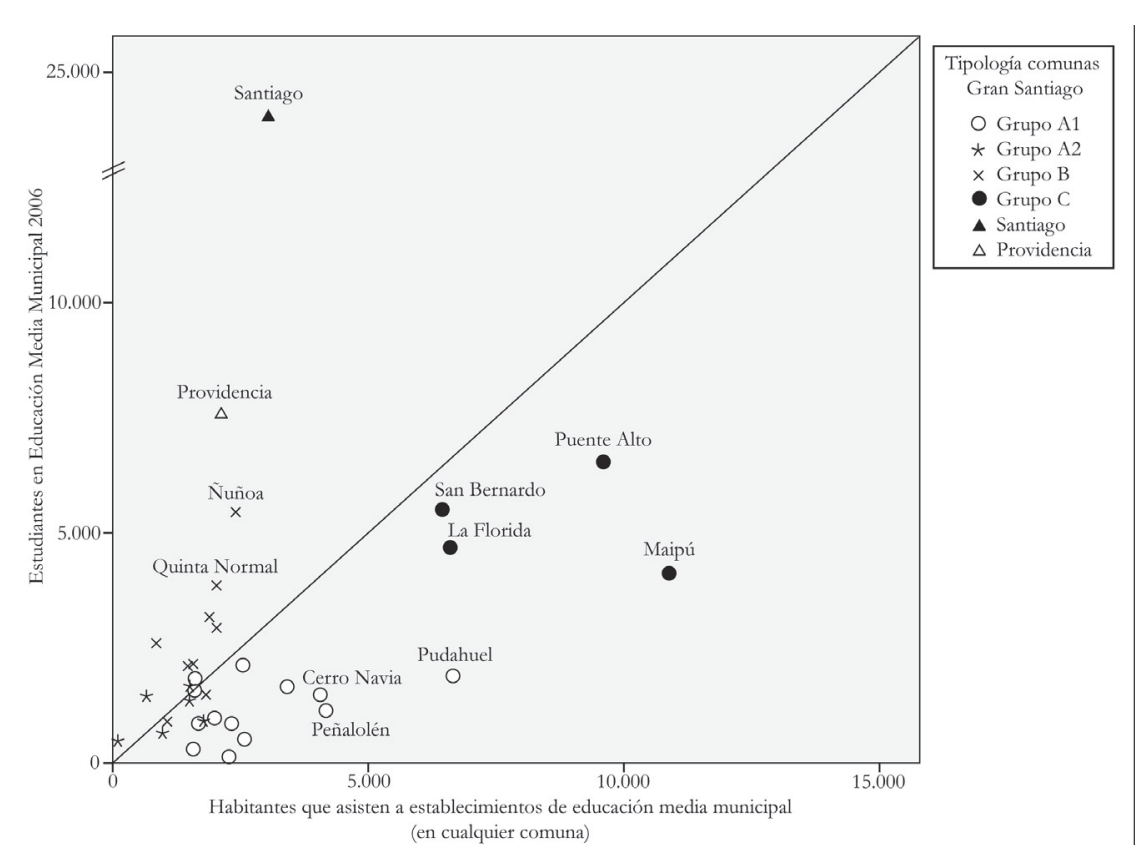

FUENTE ELABORACIÓN PROPIA A PARTIR DE MidePLAN (2006) y MiNEdUC (2007A Y 2007B).

\section{Referencias bibliográficas}

Castro Paredes, M. (2010). Códigos para el análisis de política educativa local en Chile: temas pendientes. Ensaio: Avaliação e Políticas Públicas em Educação (Río de Janeiro), 18(67), 189214. http://dx.doi.org/10.1590/S0104-40362010000200002

Consejo Asesor (2006). Informe Final del Consejo Asesor Presidencial para la Calidad de la Educación, 11 de diciembre de 2006. Disponible en www.consejoeducacion.cl/articulos/Informefinal.pdf

Donoso, S. \& Arias, Ó. (2011). Diferencias de escala en los sistemas de educación pública en Chile.

Ensaio, 19(71) (abril/junio), 283-307. 
Donoso, S. \& Hawes, G. (2002). Eficiencia escolar y diferencias socioeconómicas: a propósito de los resultados de las pruebas de Medición de la Calidad de la Educación en Chile. Educação e Pesquisa, 28(2), 25-40.

Donoso, S. \& Schmal, R. (2009). Introducción a la Economía de la Educación: El fenómeno educativo y su connotación económica. Talca: Editorial Universidad de Talca.

Donoso, S. (2006). Financiamiento y gestión de la educación pública chilena de los años 90. Cadernos de Pesquisa, 36(127) (enero-abril), 151-172. http://dx.doi.org/10.1590/S010015742006000100007

Donoso, S., Arias, Ó., Cancino, V., Oyarzún, C. (2010). Desarrollo educacional y territorio en la región de Aysén. En: J. Gairín \& D. Castro (Eds.), Desarrollo educativo al servicio del desarrollo social (pp. 71-90). Santiago: Universidad Autónoma de Barcelona/Fundación Fidecap.

Hsieh, Ch. \& Urquiola, M. (2002). When School Competes, How They Compete? An assessment of Chile's nationwide school voucher program. Washington, D.C.: World Bank's Development Group.

Hsieh, Ch. \& Urquiola, M. (2006). The effects of generalized school choice on achievement and stratification: Evidence from Chile's voucher program. Journal of Public Economics, 90, 14771503.

Ministerio de Educación (Mineduc), Chile (2007a). Tabla de directorio de establecimientos. Santiago: Autor.

Ministerio de Educación (Mineduc), Chile (2007b). Tabla de matrícula. Santiago: Autor.

Ministerio de Educación (Mineduc), Chile (2008). La educación en cifras. Santiago: Autor.

Ministerio de Educación (Mineduc), Chile (2009). Sistema de Medición de Calidad de la Educación (Simce), Carpetas de Prensa 2002-2009. Disponible en http://www.simce.cl/index.php.

Ministerio de Planificación y Cooperación (Mideplan), Chile (2006). Encuesta de Caracterización Socioeconómica Nacional (CASEN). Santiago: Autor.

Mizala, A. \& Romaguera, P. (2003). Regulación, incentivos y remuneraciones de los profesores en Chile. En: C. Cox (Ed.), Politicas educacionales en el cambio de siglo (pp. 519-558). Santiago: Editorial Universitaria.

Roemer, J. (1998) Igualdad de oportunidades. Isegoría, 18, 71-87. doi:10.3989/isegoria.1998.i18.146

Sapelli, C. (2006). Desafios del sistema educativo: La subvención preferencial. Serie Temas de Agenda Pública, Año 1, n. ${ }^{\circ}$ (junio). Santiago de Chile: Pontificia Universidad Católica de Chile, Vicerrectoría de Comunicaciones y Asuntos Públicos. Disponible en http://www.politicaspublicas.uc.cl/media/publicaciones/pdf/20100622170943.pdf

Simce (Sistema de Medición de Calidad de la Educación). Véase Ministerio de Educación (Mineduc), Chile (2009).

Sinim (Sistema Nacional de Información Municipal). Véase Subsecretaría de Desarrollo Regional y Administrativo, Ministerio del Interior, Chile (2007).

Subsecretaría de Desarrollo Regional y Administrativo, Ministerio del Interior, Chile (2007). Sistema Nacional de Información Municipal (Sinim). Diponible en http://www.sinim.gov.cl/

Tokman, A. (2001). Is Private Education Better? Evidence from Chile. Santiago: Banco Central de Chile.

Treviño, E. \& Donoso, F. (2010). Agrupación de escuelas para intervenciones de politica: Análisis del caso chileno. Santiago: Facultad de Educación, Universidad Diego Portales.

Valenzuela, J. P. (2006). Elementos financieros críticos relativos a la educación municipal. Revista Electrónica Agenda Pública, 5(10). 